\section{$\overline{\overline{\overline{\equiv \text { NASA }}}}$ \\ Paper \\ 3137}

August 1991

\section{$\underline{\underline{\underline{\underline{\underline{\underline{\underline{v}}}}}}}=$}

$1 N-92$

$368 / 6$

\title{
Analyses of Risks Associated With Radiation Exposure From Past Major Solar Părticle Events
}

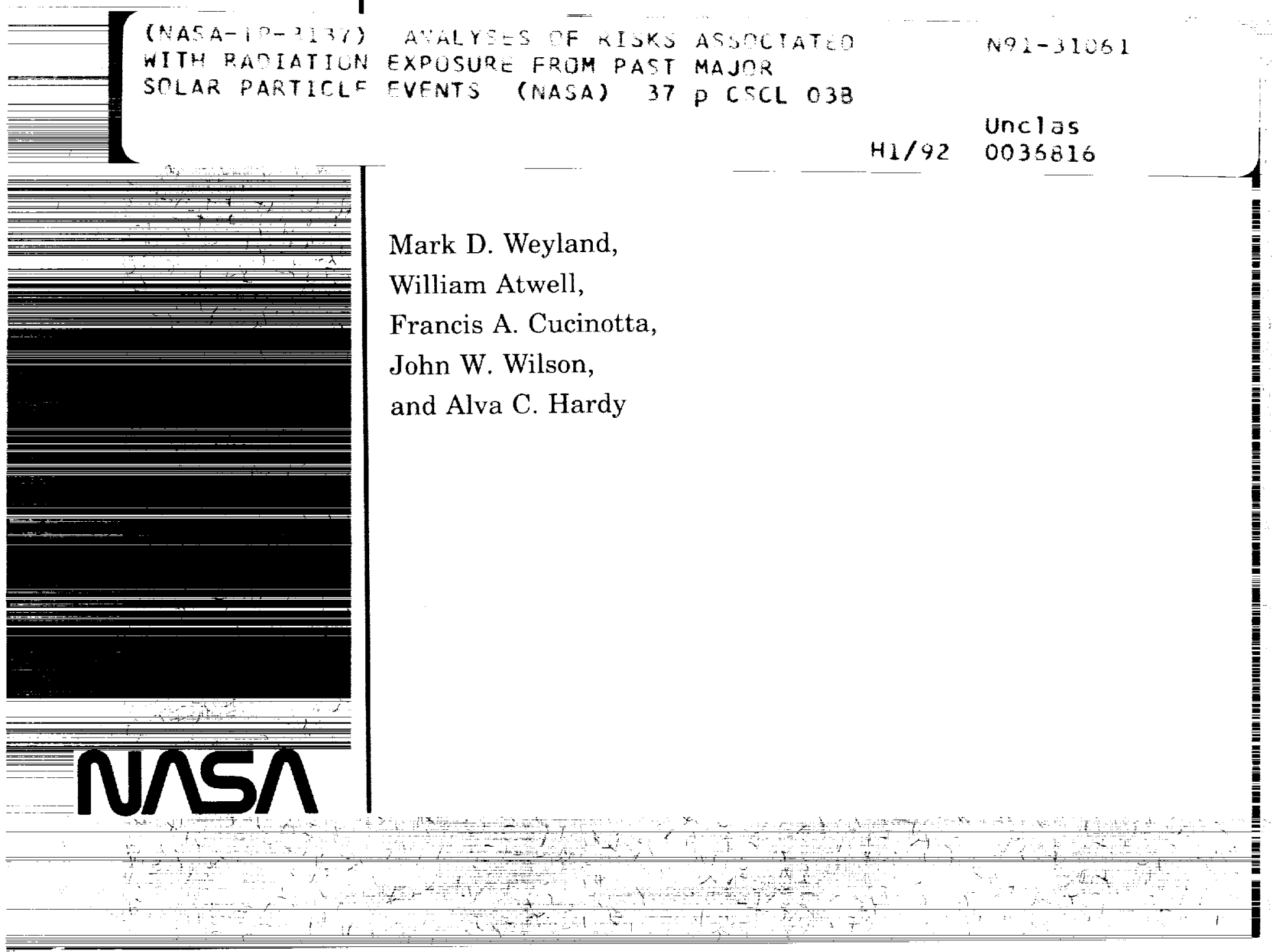


Errata

NASA Technical Paper 3137

August 1991

\title{
Analyses of Risks Associated With Radiation Exposure From Past Major Solar Particle Events
}

\author{
Weyland, M.D.; Atwell, W.; Cucinotta, F.A.; Wilson, J.W.; Hardy, A.C.
}

In the computation of excess cancers and mortalities in this paper, a conversion to SI units of two variables was inadvertently omitted. The correction lowered the risks by varying degrees of magnitude. The corrected results are given in the following tables. The secondary particle contribution has been lowered by a factor of 2 for the total risk at $10 \mathrm{~g} / \mathrm{cm}^{2}$. The conclusions remain the same with the exception of specific referenced numerical risks which have been corrected herein. The authors apologize for these errors and regret any inconvenience this omission may have caused. 



\section{NASA \\ Technical \\ Paper \\ 3137}

1991

\section{Analyses of Risks Associated With Radiation Exposure From Past Major Solar Particle Events}

Mark D. Weyland and William Atwell

Rockwell International

Houston, Texas

Francis A. Cucinotta and John W. Wilson

Langley Research Center Hampton, Virginia

Alva C. Hardy Lyndon B. Johnson Space Center Houston, Texas 



\section{ABSTRACT}

This study investigated radiation exposures and cancer induction/mortality risks for several major solar particle events (SPE's). The SPE's included in this study are: February 1956, November 1960, August 1972, October 1989, and the September, August, and October 1989 events combined. The three 1989 events were treated as one since all three could affect a single lunar or Mars mission. A baryon transport code was used to propagate particles through aluminum and tissue shield materials. This study utilized a free space environment for all calculations. Results show the 30-day blood forming organs (BFO) limit of 25 rem was surpassed by all five events using $10 \mathrm{~g} / \mathrm{cm}^{2}$ of shielding. The BFO limit is based on a depth dose of $5 \mathrm{~cm}$ of tissue, while this study utilized a more detailed shield distribution of the blood forming organs. A comparison between the $5 \mathrm{~cm}$ depth dose and the dose found using the BFO shield distribution shows the $5 \mathrm{~cm}$ depth value slightly higher than the BFO dose. The annual limit of 50 rem was exceeded by the August 1972, October 1989, and the three combined 1989 events with $5 \mathrm{~g} / \mathrm{cm}^{2}$ of shielding. Cancer mortality risks ranged from 1.5 to $17 \%$ at $1 \mathrm{~g} / \mathrm{cm}^{2}$ and 0.5 to $1.1 \%$ behind $10 \mathrm{~g} / \mathrm{cm}^{2}$ of shielding for the five events. These ranges correspond to those for a 45 year old male. It is shown that secondary particles comprise about $1 / 3$ of the total risk at $10 \mathrm{~g} / \mathrm{cm}^{2}$ of shielding. Utilizing a computerized Space Shuttle shielding model to represent a typical spacecraft configuration in free space at the August 1972 SPE, average crew doses exceeded the BFO dose limit. 


\section{INTRODUCTION}

The primary radiation concerns during manned space flights are the trapped radiation belts surrounding the earth, galactic cosmic rays (GCR), and solar particle events (SPE). While trapped radiations are limited in spatial extent and can largely be avoided, the low intensity GCR's are always present and the SPE's are potentially of high intensity although limited in time and spatially unavoidable except deep within the Earth's magnetic field. A large SPE during a lunar or Mars mission, where the spacecraft is not protected by the Earth's geomagnetic field, could have acute as well as chronic adverse health effects on improperly shielded crewmembers (Townsend et al., 1990). The effects of protons, the primary radiation associated with an SPE, on the body are subject to uncertaintes due to the high Linear Energy Transfer (LET) secondary particles produced from target fragmentation (Cucinotta, 1991).

This study investigated human organ radiation exposures behind different depths of aluminum for some past major SPE's in free space. The doses used herein were derived explicitly from the SPE's and exclude the GCR contribution which would be present and significant on long duration exploratory class missions. The skin, eye, and BFO doses were compared with the limits currently used by NASA. Cancer risks were attained for each organ and summed to give a total risk of excess cancers and cancer mortalities from these SPE's. To illustrate the importance of secondary particles, risks were calculated and shown for both the primary and secondary particles for one of the flares. Doses and risks were also obtained for two dosimeter locations aboard the current Space Shuttle, giving a complex and realistic example differing from simple slab geometries. This study could be used as baseline data in determining a safe and reasonable thickness of shielding for a manned space exploration vehicle and an associated "storm shelter."

\section{BACKGROUND}

The energetic charged particles originating from a solar particle event were propagated through aluminum and tissue shielding material using the code BRYNTRN (Wilson, et al., 1989). BRYNTRN is a baryon transport model which utilizes the Boltzmann equation in a straight ahead 
approximation. The transport code BRYNTRN was linked to the Computerized Anatomical Man (CAM) model (Billings, Yucker, 1973) in order to obtain organ doses behind various thicknesses of aluminum shielding. The CAM model was developed in the early 1970's, and with updates remains as one of the best geometrical and anthropomorphically correct computer models available. It is based on $50^{\text {th }}$ percentile standing U.S. Air Force man.

Risks were assigned to each organ based mostly on data collected in a report to the National Institutes of Health (NIH) concerning radioepidemiological tables (Rall, et al., 1985). The risk coefficients used by the NIH working group could have substantial uncertainties associated with the age- and sex-specific values (NCRP 98, 1989). One reason for this uncertainty stems from transforming mortality rates to incidence rates based on vital statistics data (NCRP 98, 1989). The linear quadratic (L-Q) equation used in the National Council on Radiation Protection and Measurements report No. 98 (NCRP 98), was derived by scaling the linear Biological Effects of Ionizing Radiation (BEIR) coefficients (NAS / NRC, 1980) by a factor related to low or high doses at low dose rates. The NCRP 98 committee defines acute and protracted doses using 5 $\mathrm{rad} /$ day as a dividing line. A dose received at a rate greater than $5 \mathrm{rad} /$ day is considered acute whereas less than $5 \mathrm{rad} /$ day is a protracted dose. All the doses discussed in this study fall under the acute situation. This factor is termed the Dose Rate Reduction Factor (DRRF) (NCRP 98, 1989). The L-Q coefficient for the acute situation is:

$$
\text { Risk coefficient }=\text { BEIR coefficient } x \frac{D}{(D R R F)}\left[1+\frac{D}{1.16}\right]
$$

where: $\mathrm{D}=$ the acute dose in gray $(\mathrm{Gy})$,

$1.16 \mathrm{~Gy}=$ the assumed cross-over dose point for which the dose-squared contribution equals the linear contribution (Rall et al., 1985).

The assumed DRRF is 2.5 according to the NCRP 98. These coefficients were then used to express predicted lifetime risks and mortalities from cancer among 1000 persons, both age- and sex-specific. An L-Q relationship was used for all organs excluding the thyroid, where a linear relationship exists. The risks for varying acute organ doses could be obtained from the NCRP 98 
tables with the following equation:

$$
E_{n}=E_{T} \frac{\left[D_{n} \times\left[1+\frac{D_{n}}{1.16}\right]\right]}{\left[D_{T} \times\left[1+\frac{D_{T}}{1.16}\right]\right]}
$$

where: $D_{n}=$ the new dose

$E_{n}=$ excess cancers for the new dose

$\mathrm{D}_{\mathrm{T}}=$ dose used in NCRP 98 tables

$\mathrm{E}_{\mathrm{T}}=$ specific cancer excess associated with dose $\mathrm{D}_{\mathrm{T}}$.

The lifetime radiation risks computed in this report were representative of cancer sites in which the NIH Working Group provided risk coefficients (Rall, et al., 1985) and for "other" cancers. It has been shown that most cancers can be caused by radiation; however, there is little or no evidence of radiation causing chronic lymphocytic (CL) leukemia (NCRP 98, 1989). The leukemia risks given herein do not include this type of malignancy and will be designated as the "sum of non-CL leukemia." One excess cancer per million person-year-rad was assigned for "other" sites by the BEIR committee and coefficients were then computed by assuming them to be proportional to those for deaths from all cancers, except leukemia, in the atomic bomb survivors of both sexes combined (NCRP 98, 1989). The organs of reference used in the "other" site category are tumors of the oral cavity, rectum, gallbladder, uterine corpus, uterine cervix, ovary, brain, bone, connective tissue, prostate and testis, and melanoma, lymphoma, and Hodgkin's disease (NCRP 98, 1989).

This study used dose equivalent numbers in place of absorbed doses to account for the higher LET values associated with space radiation. It should be noted that the risks shown in this study are subject to significant uncertainty due to the many assumptions used in computing final values. These risks are best estimates using available data and methods for risk projections. 


\section{PROCEDURE}

The code BRYNTRN was run for six past significant SPE's including February 1956, November 1960, August 1972, August 1989, September 1989, and October 1989. The 1989 events were combined for one case since all three SPE's could have affected a single lunar or Mars mission. The October 1989 flare was also used singularly because of its size. The code was modified to transport SPE particles through seven different depths of aluminum and 16 different thicknesses of tissue $\left(\mathrm{H}_{2} \mathrm{O}\right.$ in the code). BRYNTRN was set up such that the particles were transported through the 16 depths of tissue following the propagation through each thickness of aluminum. Organ doses were interpolated using these data in conjunction with organ shield distributions from the CAM model. The shield distributions are obtained by selecting a dose point in the organ and ray tracing isotropically to outside the body. These organ doses could then be applied to equation 2.2 to obtain organ-, age-, and sex-specific risks. A short code, RISK, was written to accomplish this task. To show the contribution of secondary particles, the output of BRYNTRN was modified to give doses from both secondary and primary particles. Following this, the same procedure described above was followed to obtain risks. This was done only for the August 1972 flare. To show organ doses and risks in a situation other than slab geometries, two dosimeter locations (DLOC) on the current Space Shuttle were chosen, historically the points with the highest and lowest dose readouts, and put into free space for the August 1972 flare. These results were obtained by interpolating the BRYNTRN output twice, first through the Shuttle using its shield distribution model, and then through the body. Risks were then found following the methodology described above.

\section{RESULTS}

Selected organ doses and dose equivalents vs. thickness of aluminum shielding are shown in figures $1-10$. These plots give a good indication of where additional shielding produces only a small decrease in doses. A comparison of the BFO, skin, and eye doses with the limits is given in tables $1-5$. One should recall that all the doses and risks shown herein are representative 
of free space, outside the earth's magnetosphere. As can be seen, the BFO limit is exceeded until $10 \mathrm{~g} / \mathrm{cm}^{2}$, table 1 , for the August 1972 flare, and this does not take into account the GCR contribution. Tables 6 - 25 show the organ-, age-, and sex-specific cancer and mortality risks for the different flares behind 1 and $10 \mathrm{~g} / \mathrm{cm}^{2}$ of $\mathrm{Al}$ shielding. It should be noted that due to the lack of a suitable female model at the time this study was conducted, the risk from the breasts was left out for the female. This is significant in that the breasts generally represent the greatest single organ cancer risk to the female and the exposure is expected to be relatively high (Shavers et al., 1991). Upon completion of a female model, currently being worked on, risks will be calculated and documented. The risk contribution from secondary particles can be seen in tables $26-29$ and average approximately $33 \%$ of the total risk at $10 \mathrm{~g} / \mathrm{cm}^{2}$ of Al shielding. Tables $30-34$ show the doses and associated risks at dosimeter locations (DLOC) \#1 and \#2 on the current Space Shuttle in free space for the August 1972 event.

\section{CONCLUSIONS}

This study investigated organ doses from many different perspectives. The current NASA limits were compared with the BFO, skin, and eye computed doses. It can be seen that for each of the SPE's, the BFO doses exceeded the current 30-day limit of $25 \mathrm{rem}$ using $10 \mathrm{~g} / \mathrm{cm}^{2}$ of aluminum shielding. It should be stated that the limits for Space Exploration Initiative (SEI) class missions could vary from current limits depending on other nominal risks associated with these flights and the feasibility of staying below a predetermined dose limit. The annual limit, 50 rem, was surpassed by the August 1972, October 1989, and the three 1989 events combined behind $5 \mathrm{~g} / \mathrm{cm}^{2}$ of aluminum. Graphs of doses and dose equivalents for other organs vs. shield thickness are shown in figures 1 - 10. These figures give a representation of where more shielding yields small changes in dose for each event. This appears to be between 10 and $15 \mathrm{~g} / \mathrm{cm}^{2}$ on average for the flares used in this study. Tables 6 - 25 display age- and sex-specific risks based on the organ doses from each SPE. These risks were calculated behind 1 and $10 \mathrm{~g} / \mathrm{cm}^{2}$ of shielding. The risks are displayed as excess cancers and cancer mortalities per 1000 people. For the three combined 
1989 events behind $10 \mathrm{~g} / \mathrm{cm}^{2}$ of shielding, a 25 year old male would have approximately a $2.5 \%$ risk of obtaining a fatal cancer. This doesn't take into account the GCR contribution which would add to this risk. To show the contribution of secondary particles, tables 26 - 29 show excess cancers and cancer mortalities for a 25 year old male due to both primary protons and secondary particles. The secondary particle contribution is approximately $1 / 3$ of the total risk at $10 \mathrm{~g} / \mathrm{cm}^{2}$. The last set of tables, 30 - 34, show organ doses and their respective risks at two representative dosimeter locations on the current Space Shuttle for the August 1972 event in free space. It can be seen that the annual limit of $50 \mathrm{rem}$ for the BFO was surpassed at DLOC $\# 2$ and was approached at DLOC\#1. It should be noted that these two dosimeter locations are historically the highest (\#2) and lowest (\#1) dose points recorded during flights. The measured crewmember exposures have historically averaged approximately $110 \%$ of the DLOC \#1 dose.

The flare doses behind varying depths of Al obtained with BRYNTRN correlated well with previously published values. As a reminder, these doses and risks do not include the additional exposure from GCR's which would be significant on an SEI mission. The results of this study give a detailed assessment of risks associated with major SPE's. The question of how much shielding is required for a "storm shelter" will be based on an amount equal to or greater than that shielding required to keep all doses at or below a level such that the mortality risk to the crewmembers stays below a predetermined value (e.g., 3-5\%). The total radiation risk would require evaluations of contributions from the GCR as well as any onboard sources such as nuclear power. This does not exclude the As Low As is Reasonably Achievable (ALARA) principle, however; and it should be stressed that radiation limits of any kind, risk, or dose are established as maximums and not quotas. 


\section{REFERENCES}

Wilson, John W.; Townsend, Lawrence W.; Nealy, John E.; Chun, Sang Y.; Hong, B.S.; Buck, Warren W.; Lamkin, S.L.; Ganapol, Barry D.; Khan, Ferdous; and Cucinotta, Francis A.: BRYNTRN: A Baryon Transport Model. NASA TP-2887, 1989.

National Council on Radiation Protection and Measurements (NCRP): Guidance on Radiation Received in Space Activities. NCRP Report No. 98; Bethesda, MD, 1989.

U.S. Department of Health and Human Services - National Institutes of Health (NIH): Report of the National Institutes of Health Ad Hoc Working Group to Develop Radioepidemiological Tables. NIH Publication No. 85-2748; U.S. Government Printing Office, Washington, D.C., 1985.

National Academy of Sciences/National Research Council (NAS/NRC): Assigned Share for Radiation as a Cause of Cancer: Review of Assumptions and Methods for Radioepidemiologic Tables. NAS/NRC (1984); National Academy Press, Washington, D.C., 1984.

Cucinotta, Francis A.; Katz, Robert; Wilson, John W.; Townsend, Lawrence W.; Shinn, Judy; Hajnal, Ferenc: Biological Effectiveness of High Energy Protons: Target Fragmentation. Submitted to Radiation Research; Reston, VA., 1991.

Shavers, Mark; Poston, John W.; Atwell, William; Hardy, Alva C.; Wilson, John W.: A Preliminary Calculation of Solar Cosmic Ray Dose to the Female Breast in Space Missions. Proposed NASA TM, 1991.

Townsend, Lawrence W.; Wilson, John W.; Shinn, Judy L.; Curtis, S.B.: Human Exposure to Large Solar Particle Events in Space. Presented to NATO, 1990.

Townsend, Lawrence W.; Nealy, John E.; Wilson, John W; Atwell, William: Large Solar Flare Radiation Shielding Requirements for Manned Interplanetary Missions. Journal of Spacecraft and 
Rockets; 26-2 pp.126-128; Washington, D.C. 1989.

Townsend, Lawrence W.; Shinn, Judy L.; Wilson, John W.: Interplanetary Crew Exposure Estimates for the August 1972 and October 1989 Solar Particle Events. Radiation Research (In Press); Reston, VA., 1991. 


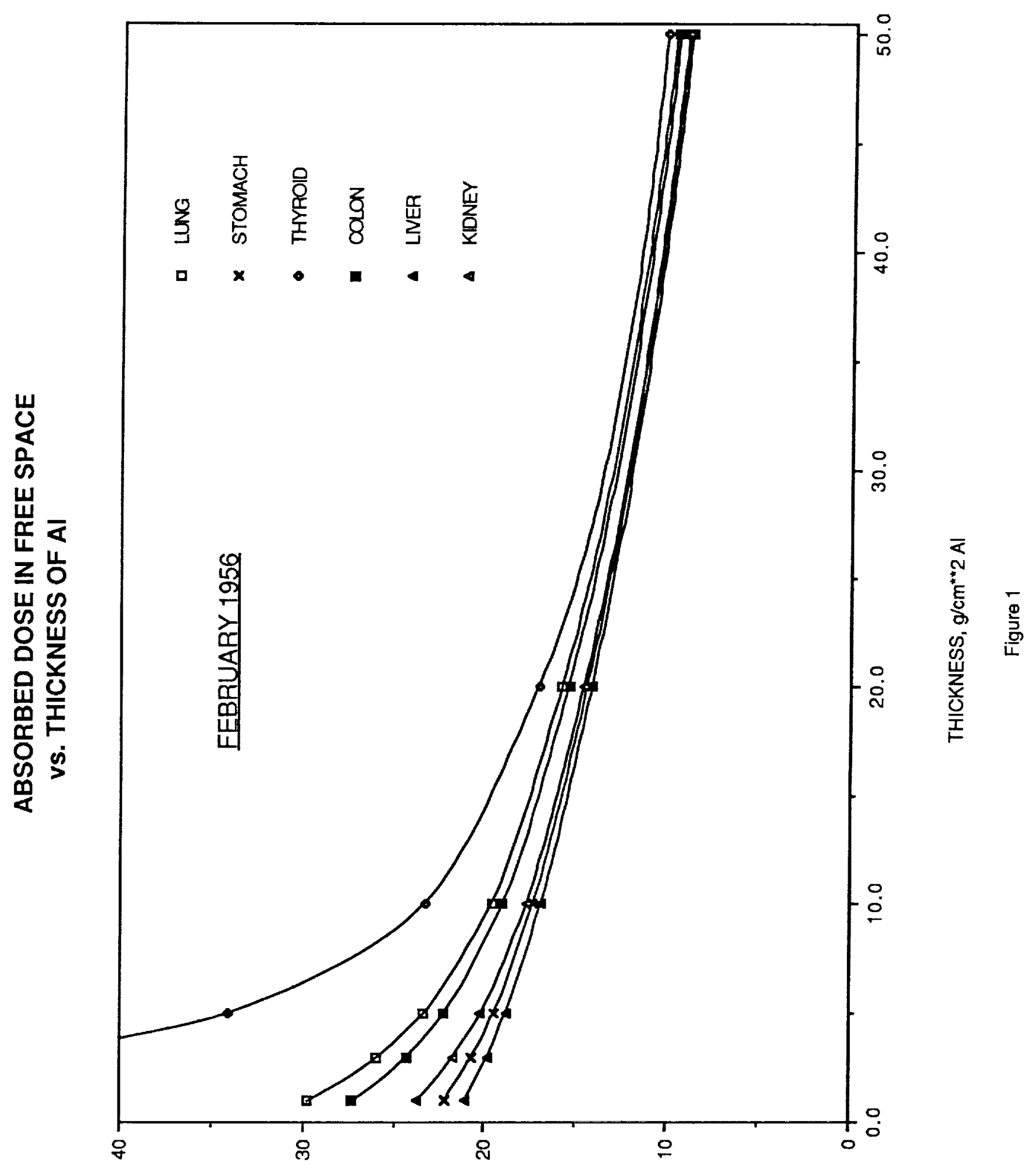

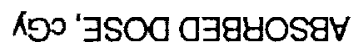




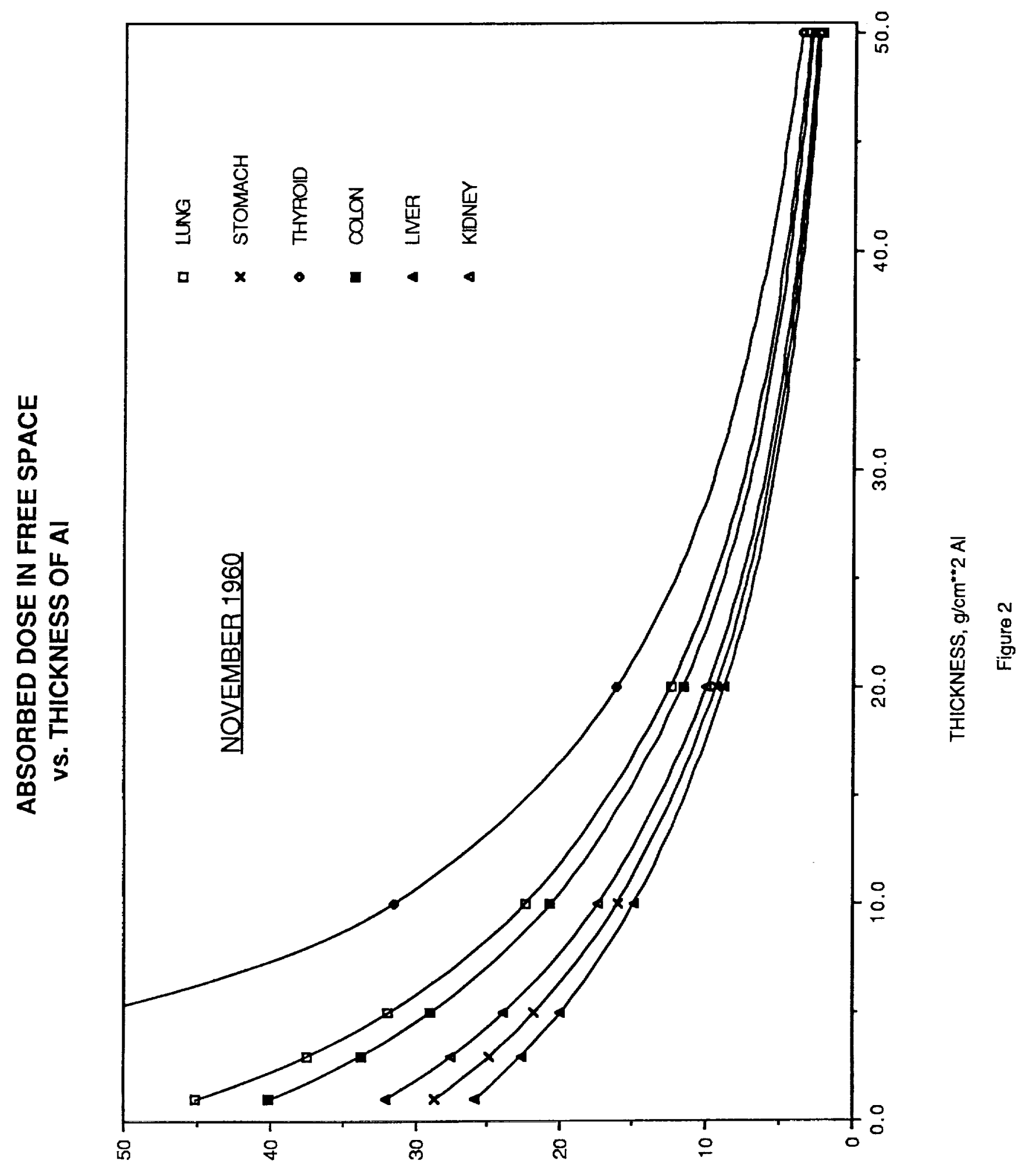

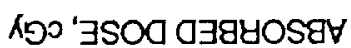




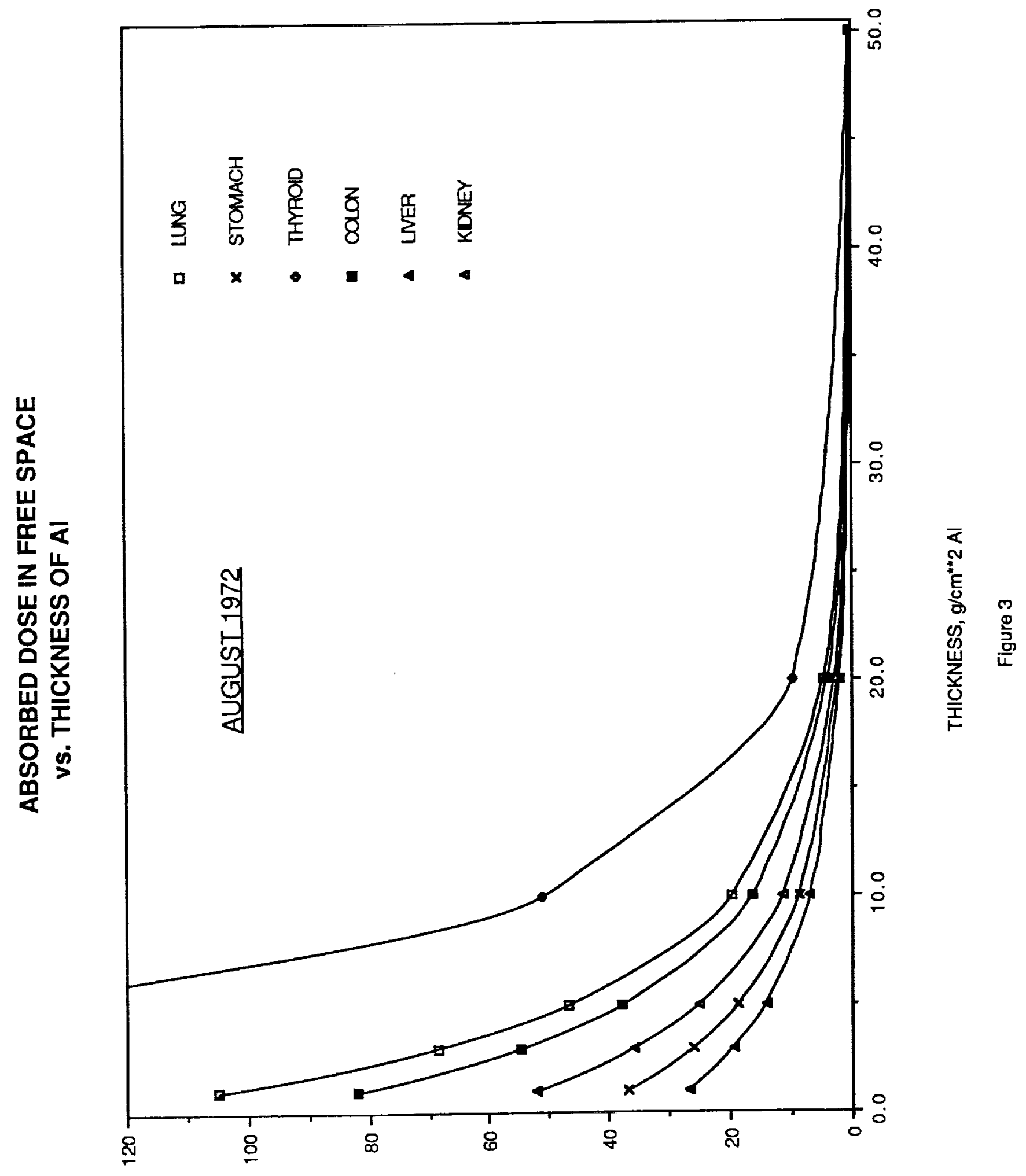

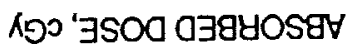




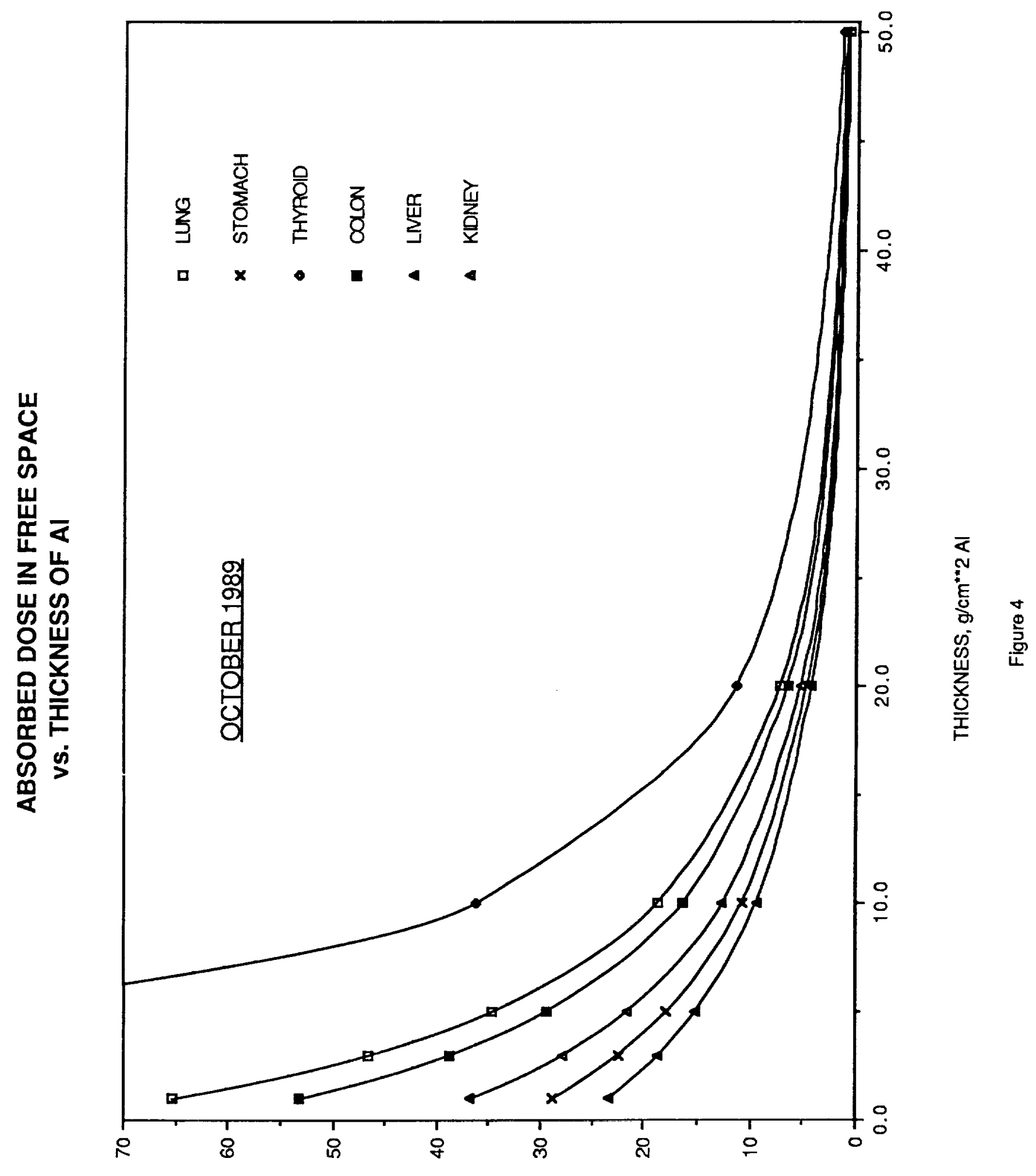

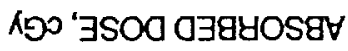




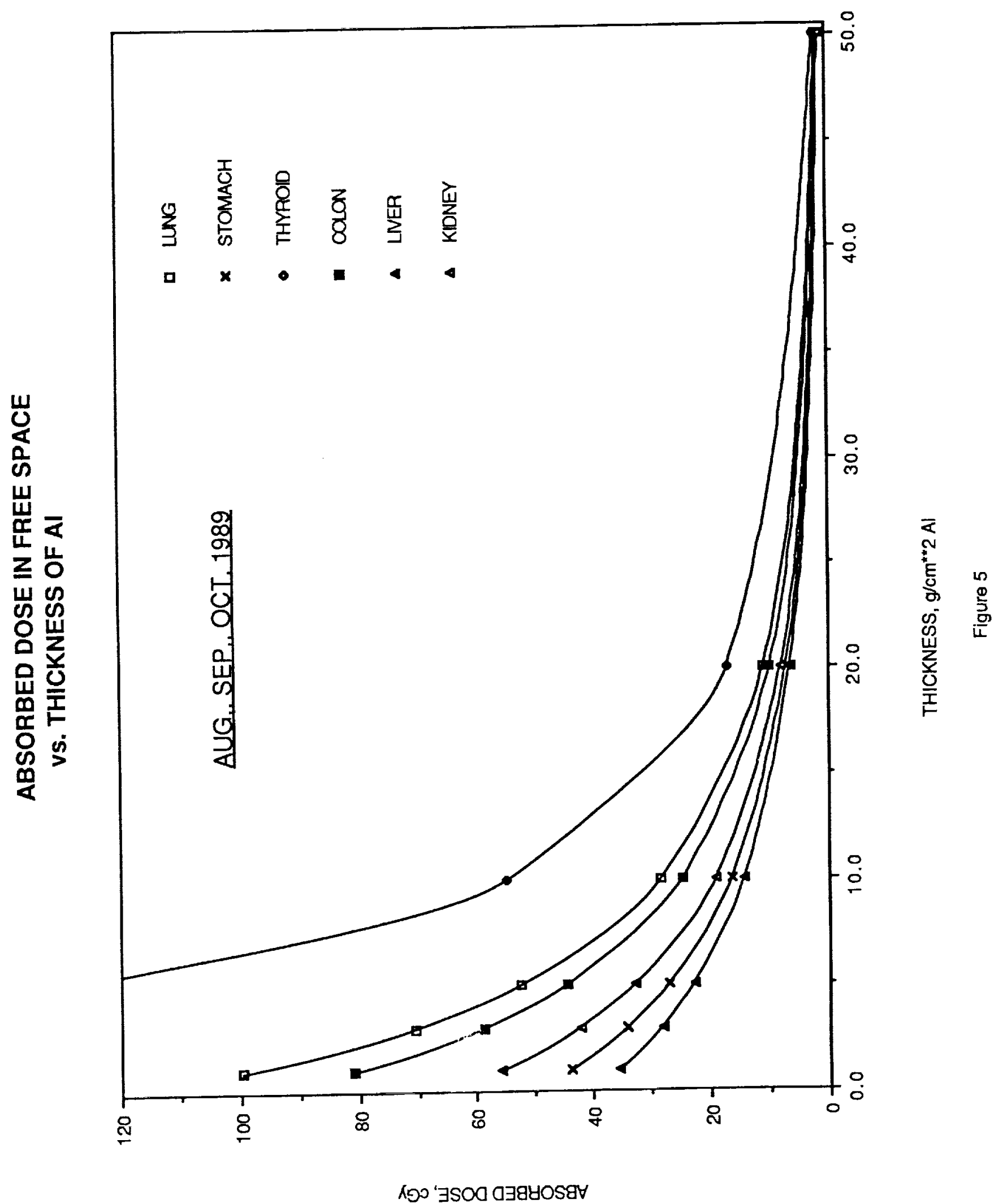




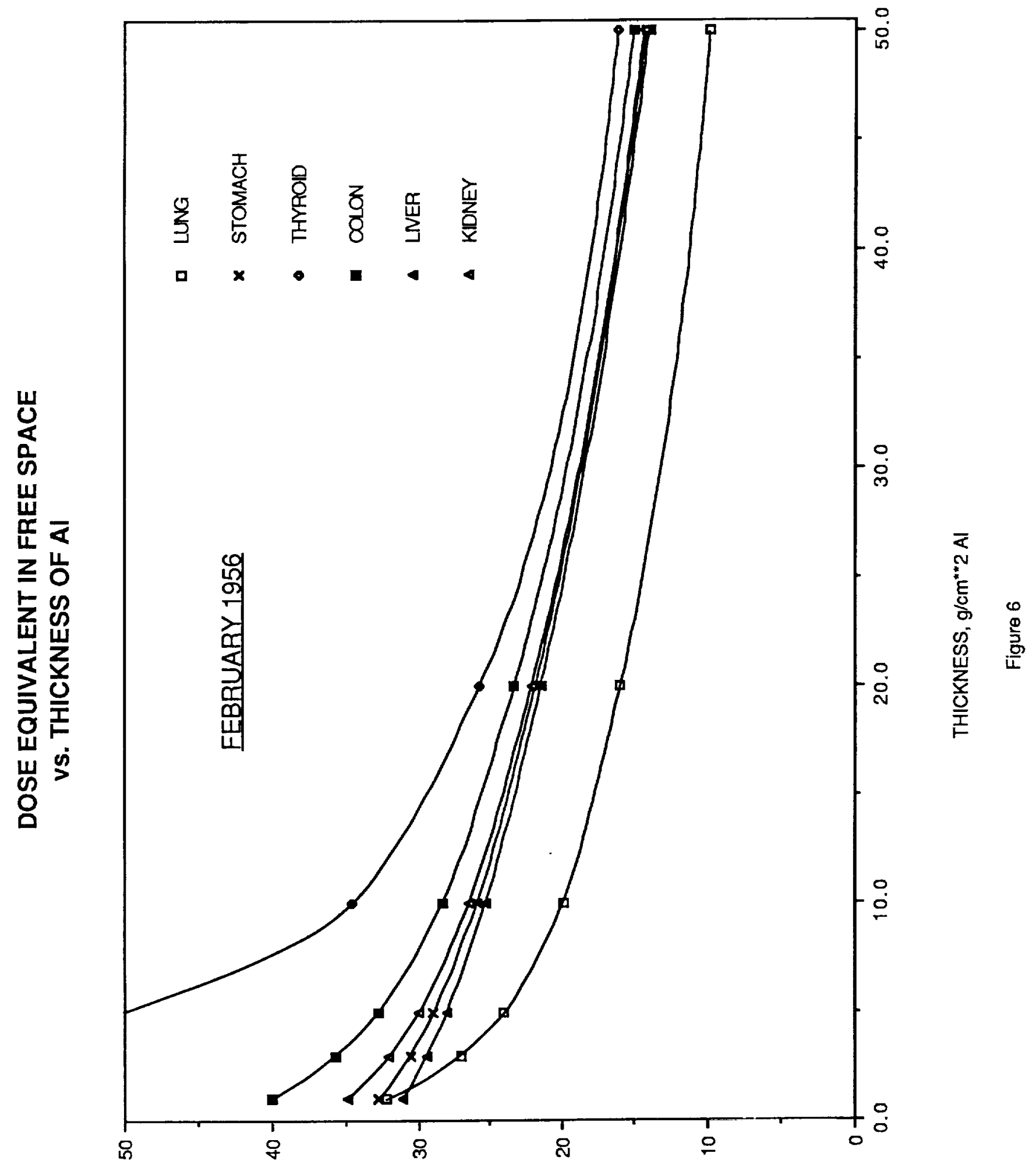

AST 'INJI $\forall A I \cap O \exists \exists S O O$ 


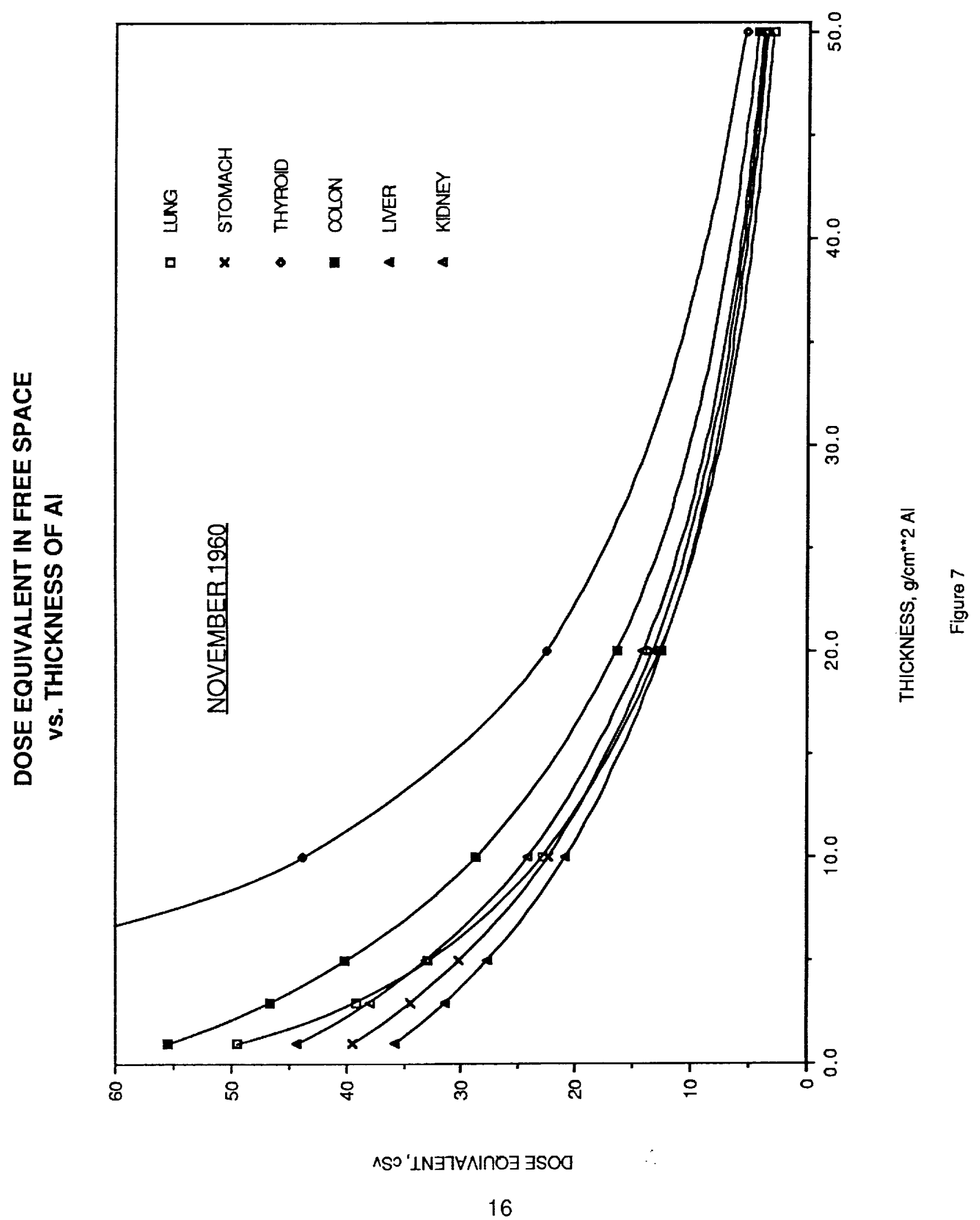




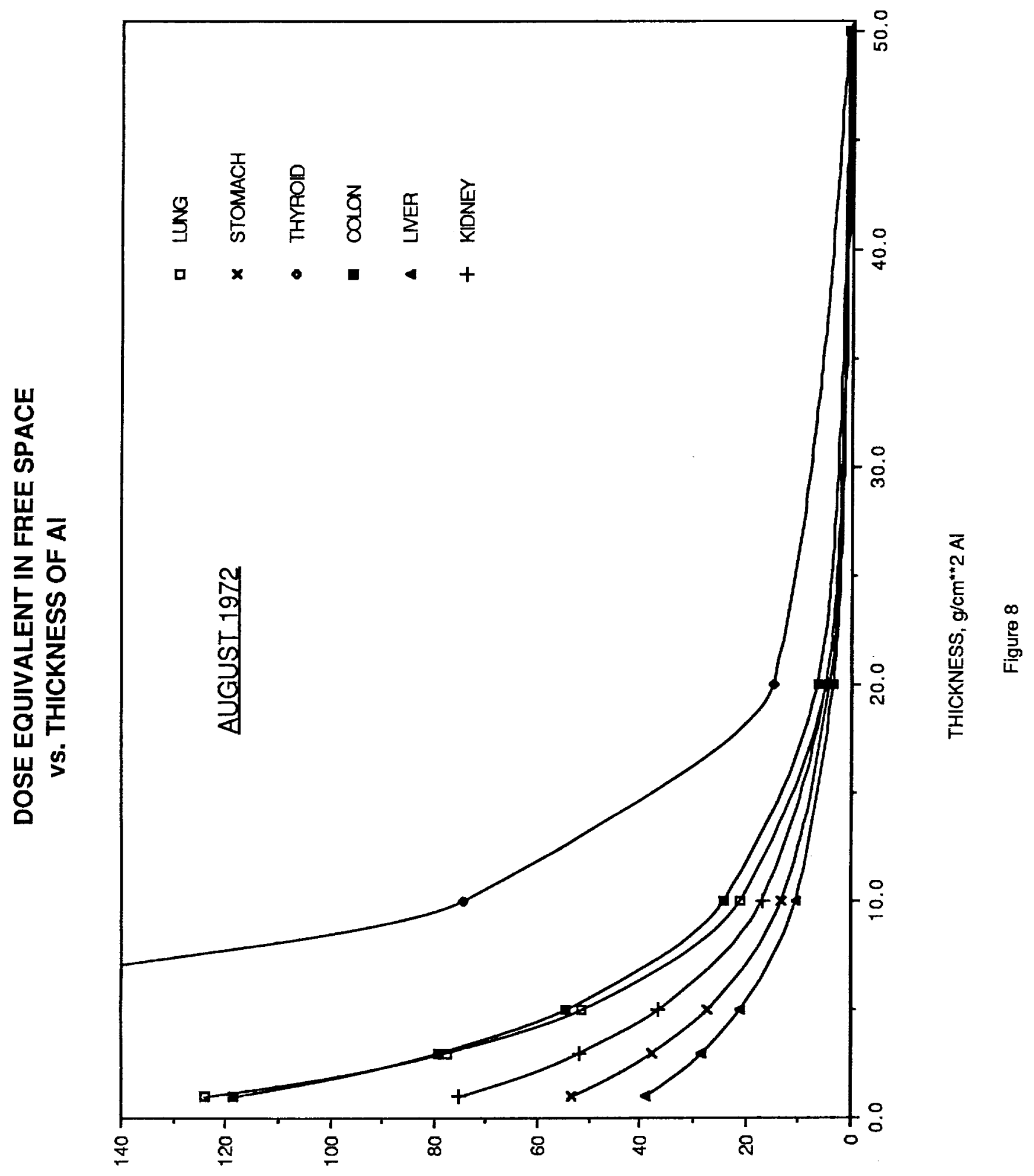

ASO 'INGTHAIกOت 


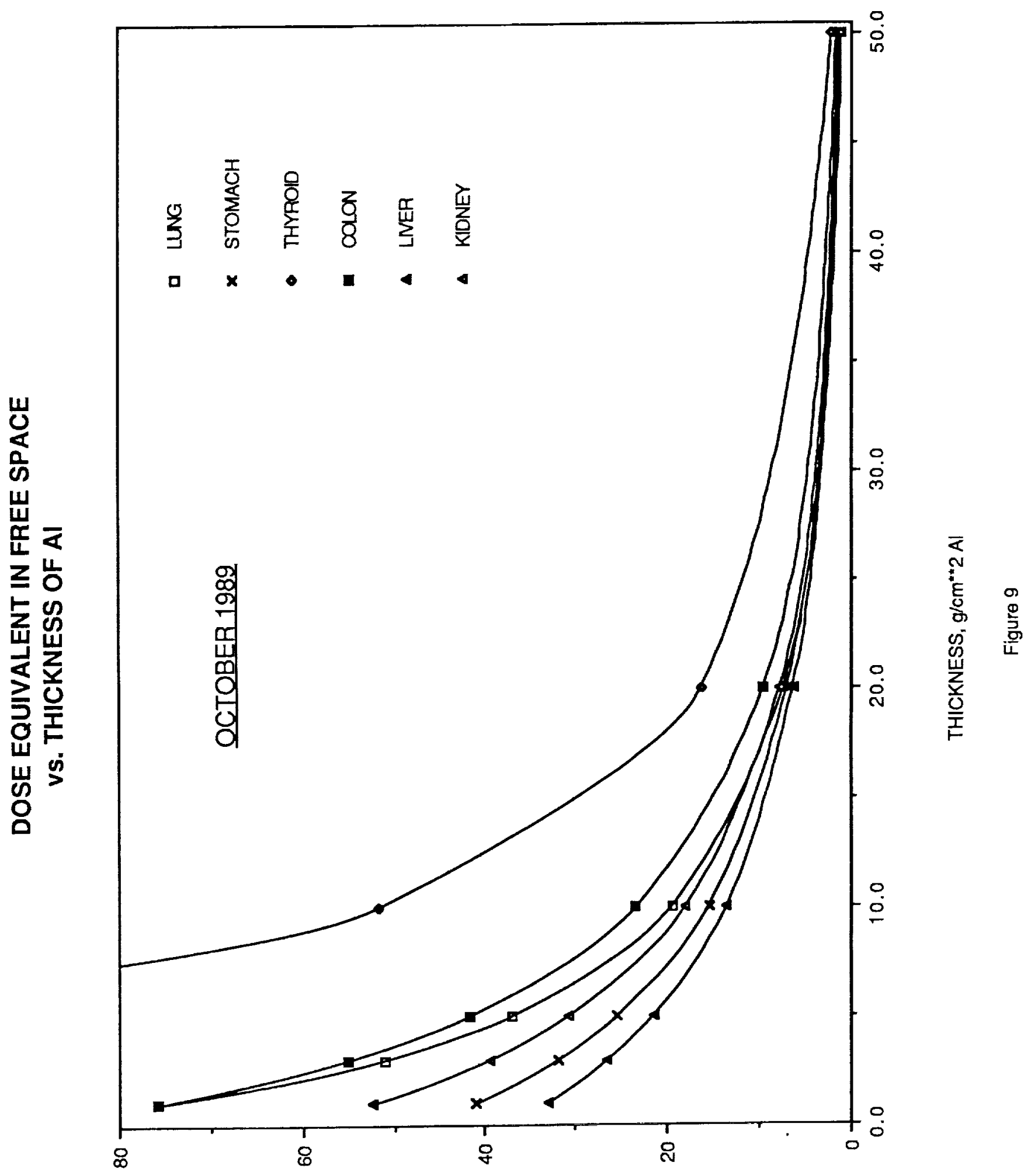

ASO 'INJTVAIกOJ JSOO 


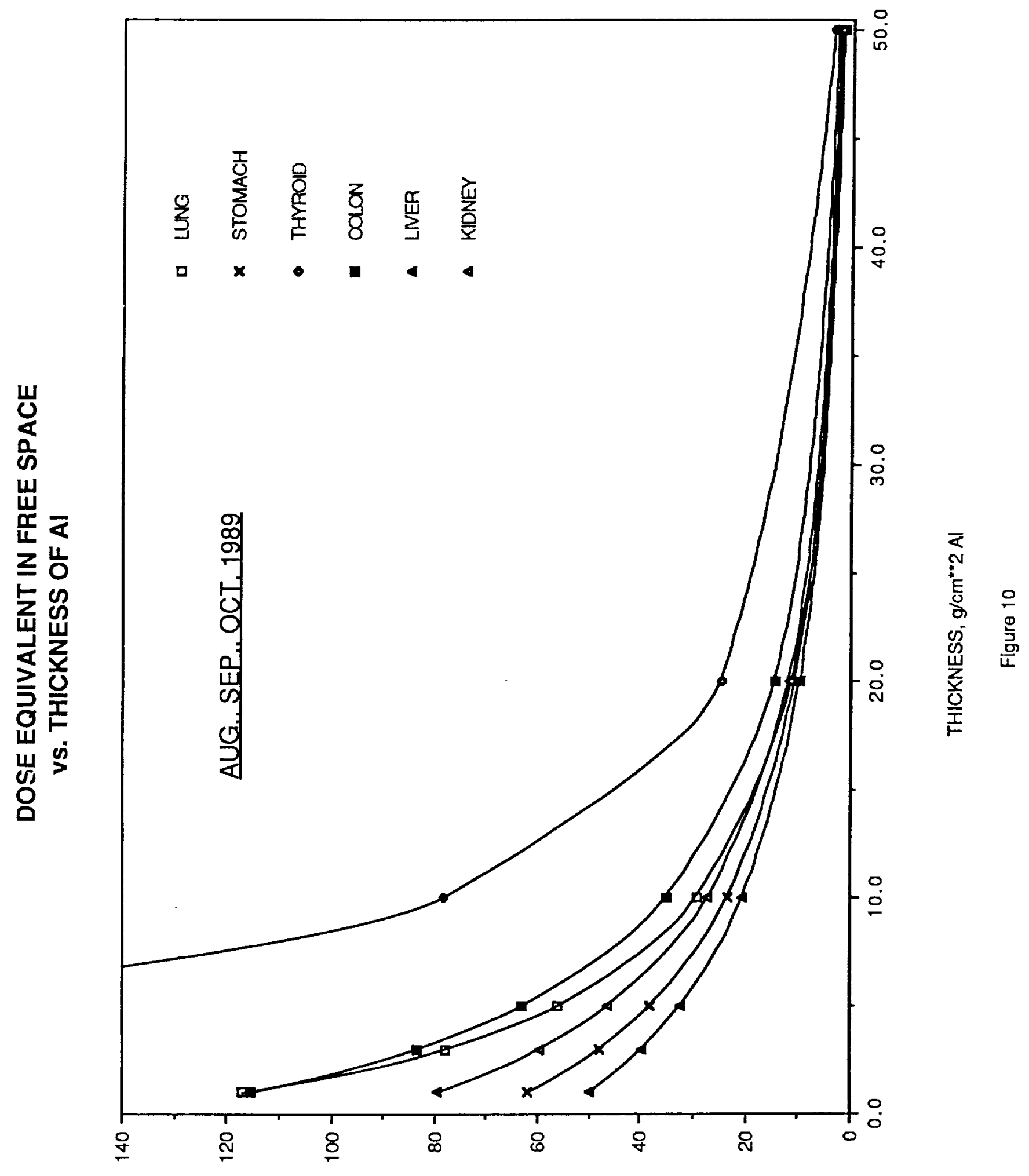

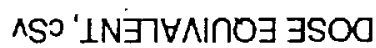


Table 1 DOSE EQUIVALENTS (REM) FROM THE AUGUST 1972 SPE IN FREE SPACE

\begin{tabular}{|c|c|c|c|c|c|c|c|}
\hline \multirow[b]{2}{*}{ ORGAN } & \multicolumn{5}{|c|}{ ALUMINUM SHIELD THICKNESS, $\mathrm{g} / \mathrm{cm}^{* *} 2$} & \multirow[b]{2}{*}{$\begin{array}{c}30 \text { DAY LIMIT } \\
\text { (REM) }\end{array}$} & \multirow[b]{2}{*}{$\begin{array}{c}\text { ANNUAL LIMIT } \\
\text { (REM) }\end{array}$} \\
\hline & 1 & 3 & 5 & 10 & 20 & & \\
\hline BFO & 183.2 & 113.5 & 74.80 & 30.86 & 7.713 & 25 & 50 \\
\hline SKIN & 1095 & 347.6 & 194.4 & 63.67 & 13.37 & 150 & 300 \\
\hline EYE & 1461 & 617.0 & 336.3 & 105.3 & 19.83 & 100 & 200 \\
\hline
\end{tabular}

Table 2 DOSE EQUIVALENTS (REM) FROM THE FEBRUARY 1956 SPE IN FREE SPACE

\begin{tabular}{|l|c|c|c|c|c|c|c|}
\hline \multirow{2}{*}{ ORGAN } & \multicolumn{6}{|c|}{ ALUMINUM SHIELD THICKNESS, g/cm**2 } & \multicolumn{2}{|c|}{$\begin{array}{c}\text { DAY LIMIT } \\
\text { (REM) }\end{array}$} & $\begin{array}{c}\text { ANNUAL LIMIT } \\
\text { (REM) }\end{array}$ \\
\cline { 2 - 7 } & 1 & 3 & 5 & 10 & 20 & 25 & 50 \\
BFO & 47.34 & 39.73 & 35.39 & 29.62 & 24.10 & 150 & 300 \\
SKIN & 264.2 & 91.19 & 61.60 & 37.51 & 26.86 & 100 & 200 \\
EYE & 207.3 & 98.60 & 65.59 & 39.75 & 28.07 & 100 \\
\hline
\end{tabular}

Table 3 DOSE EQUIVALENTS (REM) FROM THE NOVEMBER 1960 SPE IN FREE SPACE

\begin{tabular}{|l|c|c|c|c|c|c|c|}
\hline \multirow{2}{*}{ ORGAN } & \multicolumn{6}{|c|}{ ALUMINUM SHIELD THICKNESS, $\mathrm{g} / \mathrm{cm}^{\star *} 2$} & \multicolumn{2}{|c|}{$\begin{array}{c}30 \text { DAY LIMIT } \\
\text { (REM) }\end{array}$} & $\begin{array}{c}\text { ANNUAL LIMIT } \\
\text { (REM) }\end{array}$ \\
\cline { 2 - 7 } & 1 & 3 & 5 & 10 & 20 & 25 & 50 \\
BFO & 69.78 & 54.44 & 45.48 & 31.86 & 17.91 & 150 & 300 \\
SKIN & 4368 & 234.7 & 95.60 & 45.53 & 23.26 & 100 & 200 \\
EYE & 689.1 & 177.8 & 101.9 & 54.85 & 27.37 & 100 \\
\hline
\end{tabular}

Table 4 DOSE EQUIVALENTS (REM) FROM THE OCTOBER 1989 SPE IN FREE SPACE

\begin{tabular}{|c|c|c|c|c|c|c|c|}
\hline \multirow[b]{2}{*}{ ORGAN } & \multicolumn{5}{|c|}{ ALUMINUM SHIELD THICKNESS, $\mathrm{g} / \mathrm{cm}^{\star *} 2$} & \multirow[b]{2}{*}{$\begin{array}{c}30 \text { DAY LIMIT } \\
\text { (REM) }\end{array}$} & \multirow[b]{2}{*}{$\begin{array}{c}\text { ANNUAL LIMIT } \\
\text { (REM) }\end{array}$} \\
\hline & 1 & 3 & 5 & 10 & 20 & & \\
\hline $\mathrm{BFO}$ & 110.4 & 73.29 & 52.76 & 27.70 & 10.84 & 25 & 50 \\
\hline SKIN & 1312 & 243.6 & 129.0 & 49.83 & 16.26 & 150 & 300 \\
\hline EYE & 997.0 & 352.1 & 190.8 & 70.51 & 20.89 & 100 & 200 \\
\hline
\end{tabular}

Table 5 DOSE EQUIVALENTS (REM) FROM THE AUGUST, SEPTEMBER, \& OCTOBER 1989 SPE'S IN FREE SPACE

\begin{tabular}{|l|c|c|c|c|c|c|c|}
\hline \multirow{2}{*}{ ORGAN } & \multicolumn{6}{|c|}{ ALUMINUM SHIELD THICKNESS, $\mathrm{g} / \mathrm{cm}^{* *} 2$} & \\
\cline { 2 - 7 } & 1 & 3 & 5 & 10 & 20 & $\begin{array}{c}30 \text { DAY LIMIT } \\
\text { (REM) }\end{array}$ & $\begin{array}{c}\text { ANNUAL LIMIT } \\
\text { (REM) }\end{array}$ \\
\hline BFO & 171.3 & 112.1 & 80.15 & 41.92 & 16.54 & 25 & 50 \\
SKIN & 2287 & 383.3 & 198.8 & 75.38 & 24.69 & 150 & 300 \\
EYE & 1725 & 546.6 & 297.2 & 107.0 & 31.65 & 100 & 200 \\
\hline
\end{tabular}


Table 6

FEBRUARY 1956 SPE, EXCESS CANCERS PER 1000 PEOPLE BEHIND $1 \mathrm{~g} / \mathrm{cm}^{* * 2}$ OF AI

\begin{tabular}{|ll|c|c|c|c|c|c|}
\hline SEX, AGE @ EXP & LUNG & ESOPHAGUS & STOMACH & COLON & LIVER & PANCREAS \\
\hline$M$ & 25 & 3.812 & 0.390 & 2.043 & 2.181 & 1.666 & 1.228 \\
$M$ & 35 & 2.567 & 0.167 & 1.041 & 0.991 & 0.543 & 0.575 \\
$M$ & 45 & 1.849 & 0.111 & 0.655 & 0.545 & 0.254 & 0.345 \\
$M$ & 55 & 1.359 & 0.167 & 0.617 & 0.545 & 0.145 & 0.384 \\
$F$ & 25 & 3.020 & 0.446 & 2.891 & 2.578 & 1.956 & 1.880 \\
$F$ & 35 & 2.491 & 0.223 & 1.696 & 1.289 & 0.833 & 0.806 \\
$F$ & 45 & 2.265 & 0.167 & 1.041 & 0.793 & 0.398 & 0.499 \\
$F$ & 55 & 1.925 & 0.223 & 0.887 & 0.744 & 0.217 & 0.499 \\
& & & & & & & \\
\cline { 2 - 8 } & & SUM(NON-CL) & KIDNEY\& & THYROID & ALLOTHER & & TOTAL \\
\cline { 2 - 8 } & LEUKEMIA & BLADDER & & CANCERS & & CANCERS \\
$M$ & 1.411 & 3.002 & 2.867 & 4.541 & & 23.14 \\
$M$ & 35 & 1.534 & 1.647 & 1.864 & 2.025 & & 12.96 \\
$M$ & 45 & 1.657 & 1.065 & 1.234 & 1.105 & & 8.822 \\
$M$ & 55 & 1.657 & 0.775 & 0.733 & 0.859 & & 7.240 \\
$F$ & 25 & 0.921 & 3.584 & 8.872 & 2.884 & & 29.03 \\
$F$ & 35 & 1.043 & 2.131 & 6.429 & 1.657 & & 18.60 \\
$F$ & 45 & 1.289 & 1.453 & 4.680 & 1.227 & & 13.81 \\
$F$ & 55 & 1.411 & 1.162 & 3.137 & 1.227 & & 11.43 \\
\hline
\end{tabular}

Table 7

FEBRUARY 1956 SPE, EXCESS CANCER MORTALITIES PER 1000 PEOPLE BEHIND $1 \mathrm{~g} / \mathrm{cm}$ **2 OF AI

\begin{tabular}{|ll|c|c|c|c|c|c|}
\hline \multicolumn{2}{|c|}{ SEX, AGE @ EXP } & LUNG & ESOPHAGUS & STOMACH & COLON & LIVER & PANCREAS \\
\hline$M$ & 25 & 3.020 & 0.334 & 1.426 & 1.140 & 1.594 & 1.151 \\
$M$ & 35 & 2.038 & 0.167 & 0.732 & 0.545 & 0.543 & 0.537 \\
$M$ & 45 & 1.472 & 0.111 & 0.463 & 0.297 & 0.254 & 0.345 \\
$M$ & 55 & 1.057 & 0.111 & 0.463 & 0.297 & 0.145 & 0.345 \\
$F$ & 25 & 2.151 & 0.334 & 2.159 & 1.289 & 1.920 & 1.765 \\
$F$ & 35 & 1.812 & 0.167 & 1.272 & 0.644 & 0.797 & 0.767 \\
$F$ & 45 & 1.623 & 0.111 & 0.771 & 0.397 & 0.362 & 0.460 \\
$F$ & 55 & 1.397 & 0.167 & 0.655 & 0.347 & 0.181 & 0.460 \\
& & & & & & & \\
\cline { 2 - 8 } & & SUM (NON-CL) & KIDNEY\& & THYROID & AL OTHER & & TOTAL \\
\cline { 2 - 8 } M & 25 & 1.105 & 0.969 & 0.463 & 1.780 & & 12.98 \\
$M$ & 35 & 1.227 & 0.581 & 0.296 & 0.798 & & 7.466 \\
$M$ & 45 & 1.350 & 0.387 & 0.193 & 0.430 & & 5.302 \\
$M$ & 55 & 1.350 & 0.291 & 0.116 & 0.368 & & 4.543 \\
$F$ & 25 & 0.675 & 1.259 & 0.810 & 1.166 & & 13.53 \\
$F$ & 35 & 0.798 & 0.775 & 0.591 & 0.675 & & 8.299 \\
$F$ & 45 & 0.921 & 0.484 & 0.424 & 0.491 & & 6.045 \\
$F$ & 55 & 1.043 & 0.387 & 0.283 & 0.491 & & 5.412 \\
\hline
\end{tabular}


Table 8

FEBRUARY 1956 SPE, EXCESS CANCERS PER 1000 PEOPLE BEHIND $10 \mathrm{~g} / \mathrm{cm}^{* * 2}$ OF Al

\begin{tabular}{|c|c|c|c|c|c|c|c|}
\hline \multicolumn{2}{|c|}{ SEX,AGE@EXP } & LUNG & ESOPHAGUS & STOMACH & COLON & LIVER & PANCREAS \\
\hline$M$ & 25 & 2.175 & 0.229 & 1.547 & 1.426 & 1.308 & 0.933 \\
\hline$M$ & 35 & 1.465 & 0.098 & 0.788 & 0.648 & 0.426 & 0.437 \\
\hline$M$ & 45 & 1.055 & 0.065 & 0.496 & 0.357 & 0.199 & 0.262 \\
\hline$M$ & 55 & 0.775 & 0.098 & 0.467 & 0.357 & 0.114 & 0.291 \\
\hline $\mathrm{F}$ & 25 & 1.723 & 0.262 & 2.190 & 1.685 & 1.535 & 1.428 \\
\hline $\mathrm{F}$ & 35 & 1.421 & 0.131 & 1.285 & 0.843 & 0.654 & 0.612 \\
\hline $\mathrm{F}$ & 45 & 1.292 & 0.098 & 0.788 & 0.519 & 0.313 & 0.379 \\
\hline \multirow[t]{2}{*}{$F$} & \multirow[t]{2}{*}{55} & 1.098 & 0.131 & 0.671 & 0.486 & 0.171 & 0.379 \\
\hline & & $\begin{array}{c}\text { SUM (NON-CL) } \\
\text { LEUKEMIA }\end{array}$ & $\begin{array}{l}\text { KIDNEY \& } \\
\text { BLADDER }\end{array}$ & THYROID & $\begin{array}{l}\text { ALL OTHER } \\
\text { CANCERS }\end{array}$ & & $\begin{array}{l}\text { TOTAL } \\
\text { CANCERS }\end{array}$ \\
\hline$M$ & 25 & 0.787 & 2.137 & 0.855 & 2.533 & & 13.93 \\
\hline$M$ & 35 & 0.856 & 1.172 & 0.556 & 1.130 & & 7.576 \\
\hline$M$ & 45 & 0.924 & 0.758 & 0.368 & 0.616 & & 5.102 \\
\hline$M$ & 55 & 0.924 & 0.552 & 0.219 & 0.479 & & 4.276 \\
\hline$F$ & 25 & 0.513 & 2.551 & 2.645 & 1.609 & & 16.14 \\
\hline$F$ & 35 & 0.582 & 1.517 & 1.917 & 0.924 & & 9.885 \\
\hline$F$ & 45 & 0.719 & 1.034 & 1.395 & 0.685 & & 7.222 \\
\hline$F$ & 55 & 0.787 & 0.827 & 0.935 & 0.685 & & 6.171 \\
\hline
\end{tabular}

Table 9

FEBRUARY 1956 SPE, EXCESS CANCER MORTALITIES PER 1000 PEOPLE BEHIND $10 \mathrm{~g} / \mathrm{cm}^{* * 2}$ OF AI

\begin{tabular}{|c|c|c|c|c|c|c|c|}
\hline \multicolumn{2}{|c|}{ SEX,AGE@EXP } & LUNG & ESOPHAGUS & STOMACH & COLON & LIVER & PANCREAS \\
\hline$M$ & 25 & 1.723 & 0.196 & 1.080 & 0.745 & 1.251 & 0.874 \\
\hline$M$ & 35 & 1.163 & 0.098 & 0.555 & 0.357 & 0.426 & 0.408 \\
\hline$M$ & 45 & 0.840 & 0.065 & 0.253 & 0.194 & 0.199 & 0.262 \\
\hline$M$ & 55 & 0.603 & 0.065 & 0.350 & 0.194 & 0.114 & 0.262 \\
\hline$F$ & 25 & 1.228 & 0.196 & 1.635 & 0.843 & 1.507 & 1.341 \\
\hline$F$ & 35 & 1.034 & 0.098 & 0.963 & 0.421 & 0.625 & 0.583 \\
\hline$F$ & 45 & 0.926 & 0.065 & 0.584 & 0.259 & 0.284 & 0.350 \\
\hline \multirow[t]{2}{*}{$F$} & 55 & 0.797 & 0.098 & 0.496 & 0.227 & 0.142 & 0.350 \\
\hline & & $\begin{array}{l}\text { SUM (NON-CL) } \\
\text { LEUKEMIA }\end{array}$ & $\begin{array}{l}\text { KIDNEY \& } \\
\text { BLADDER }\end{array}$ & THYROID & $\begin{array}{l}\text { ALL OTHER } \\
\text { CANCERS }\end{array}$ & & $\begin{array}{c}\text { TOTAL } \\
\text { MORTALITIES }\end{array}$ \\
\hline$M$ & 25 & 0.616 & 0.689 & 0.138 & 0.993 & & 8.306 \\
\hline$M$ & 35 & 0.685 & 0.414 & 0.088 & 0.445 & & 4.638 \\
\hline$M$ & 45 & 0.753 & 0.276 & 0.058 & 0.240 & & 3.237 \\
\hline$M$ & 55 & 0.753 & 0.207 & 0.035 & 0.205 & & 2.789 \\
\hline$F$ & 25 & 0.377 & 0.896 & 0.241 & 0.650 & & 8.913 \\
\hline$F$ & 35 & 0.445 & 0.552 & 0.176 & 0.377 & & 5.274 \\
\hline$F$ & 45 & 0.513 & 0.345 & 0.127 & 0.274 & & 3.727 \\
\hline$F$ & 55 & 0.582 & 0.276 & 0.084 & 0.274 & & 3.326 \\
\hline
\end{tabular}


Table 10 NOVEMBER 1960 SPE, EXCESS CANCERS PER 1000 PEOPLE BEHIND $1 \mathrm{~g} / \mathrm{cm}^{* * 2}$ OF AI

\begin{tabular}{|c|c|c|c|c|c|c|c|}
\hline \multicolumn{2}{|c|}{ SEX.AGE@EXP } & LUNG & ESOPHAGUS & STOMACH & COLON & LIVER & PANCREAS \\
\hline$M$ & 25 & 6.558 & 0.618 & 2.585 & 3.319 & 1.980 & 1.543 \\
\hline$M$ & 35 & 4.415 & $0.265^{\circ}$ & 1.317 & 1.509 & 0.646 & 0.723 \\
\hline$M$ & 45 & 3.182 & 0.176 & 0.829 & 0.830 & 0.301 & 0.434 \\
\hline M & 55 & 2.338 & 0.265 & 0.780 & 0.830 & 0.172 & 0.482 \\
\hline$F$ & 25 & 5.195 & 0.706 & 3.658 & 3.923 & 2.324 & 2.362 \\
\hline$F$ & 35 & 4.286 & 0.353 & 2.146 & 1.961 & 0.990 & 1.012 \\
\hline$F$ & 45 & 3.896 & 0.265 & 1.317 & 1.207 & 0.473 & 0.627 \\
\hline \multirow[t]{2}{*}{$F$} & \multirow[t]{2}{*}{55} & 3.312 & 0.353 & 1.122 & 1.132 & 0.258 & 0.627 \\
\hline & & $\begin{array}{c}\text { SUM (NON-CL) } \\
\text { LEUKEMIA }\end{array}$ & $\begin{array}{l}\text { KIDNEY \& } \\
\text { BLADDER }\end{array}$ & THYROID & $\begin{array}{l}\text { ALL OTHER } \\
\text { CANCERS }\end{array}$ & & $\begin{array}{c}\text { TOTAL } \\
\text { CANCERS } \\
\end{array}$ \\
\hline$M$ & 25 & 2.366 & 3.961 & 6.116 & 7.614 & & 36.66 \\
\hline$M$ & 35 & 2.572 & 2.172 & 3.977 & 3.395 & & 20.99 \\
\hline$M$ & 45 & 2.778 & 1.405 & 2.633 & 1.852 & & 14.42 \\
\hline$M$ & 55 & 2.778 & 1.022 & 1.563 & 1.440 & & 11.67 \\
\hline$F$ & 25 & 1.543 & 4.727 & 18.92 & 4.836 & & 48.20 \\
\hline$F$ & 35 & 1.749 & 2.811 & 13.71 & 2.778 & & 31.80 \\
\hline$F$ & 45 & 2.161 & 1.917 & 9.982 & 2.058 & & 23.90 \\
\hline$F$ & 55 & 2.366 & 1.533 & 6.692 & 2.058 & & 19.45 \\
\hline
\end{tabular}

Table 11 NOVEMBER 1960 SPE, EXCESS CANCER MORTALITIES PER 1000 PEOPLE BEHIND $1 \mathrm{~g} / \mathrm{cm}^{* *} 2$ OF AI

\begin{tabular}{|c|c|c|c|c|c|c|c|}
\hline \multicolumn{2}{|c|}{ SEX,AGE@EXP } & LUNG & ESOPHAGUS & STOMACH & COLON & LIVER & PANCREAS \\
\hline$M$ & 25 & 5.195 & 0.529 & 1.805 & 1.735 & 1.894 & 1.446 \\
\hline$M$ & 35 & 3.506 & 0.265 & 0.927 & 0.830 & 0.646 & 0.675 \\
\hline$M$ & 45 & 2.532 & 0.176 & 0.585 & 0.453 & 0.301 & 0.434 \\
\hline$M$ & 55 & 1.818 & 0.176 & 0.585 & 0.453 & 0.172 & 0.434 \\
\hline$F$ & 25 & 3.701 & 0.529 & 2.732 & 1.961 & 2.281 & 2.218 \\
\hline$F$ & 35 & 3.117 & 0.265 & 1.610 & 0.981 & 0.947 & 0.964 \\
\hline $\mathrm{F}$ & 45 & 2.792 & 0.176 & 0.976 & 0.603 & 0.430 & 0.578 \\
\hline \multirow[t]{2}{*}{$F$} & \multirow[t]{2}{*}{55} & 2.403 & 0.265 & 0.829 & 0.528 & 0.215 & 0.578 \\
\hline & & $\begin{array}{c}\text { SUM (NON-CL) } \\
\text { LEUKEMIA }\end{array}$ & $\begin{array}{l}\text { KIDNEY \& } \\
\text { BLADDER }\end{array}$ & THYROID & $\begin{array}{c}\text { ALL OTHER } \\
\text { CANCERS }\end{array}$ & & $\begin{array}{c}\text { TOTAL } \\
\text { MORTALITIES }\end{array}$ \\
\hline$M$ & 25 & 1.852 & 1.278 & 0.987 & 2.984 & & 19.71 \\
\hline$M$ & 35 & 2.058 & 0.767 & 0.631 & 1.338 & & 11.64 \\
\hline$M$ & 45 & 2.264 & 0.511 & 0.411 & 0.720 & & 8.387 \\
\hline$M$ & 55 & 2.264 & 0.383 & 0.247 & 0.617 & & 7.150 \\
\hline $\mathbf{F}$ & 25 & 1.132 & 1.661 & 1.728 & 1.955 & & 19.90 \\
\hline$F$ & 35 & 1.338 & 1.022 & 1.262 & 1.132 & & 12.64 \\
\hline$F$ & 45 & 1.543 & 0.639 & 0.905 & 0.823 & & 9.467 \\
\hline$F$ & 55 & 1.749 & 0.511 & 0.603 & 0.823 & & 8.505 \\
\hline
\end{tabular}


Table 12

NOVEMBER 1960 SPE, EXCESS CANCERS PER 1000 PEOPLE BEHIND $10 \mathrm{~g} / \mathrm{cm}^{* * 2}$ OF AI

\begin{tabular}{|c|c|c|c|c|c|c|c|}
\hline \multicolumn{2}{|c|}{ SEX,AGE@EXP } & LIING & ESOPHAGUS & STOMACH & COLON & LIVER & PANCREAS \\
\hline$M$ & 25 & 2.559 & 0.240 & 1.307 & 1.453 & 1.042 & 0.782 \\
\hline$M$ & 35 & 1.723 & 0.103 & 0.666 & 0.661 & 0.340 & 0.367 \\
\hline$M$ & 45 & 1.241 & 0.069 & 0.419 & 0.363 & 0.159 & 0.220 \\
\hline$M$ & 55 & 0.912 & 0.103 & 0.394 & 0.363 & 0.091 & 0.245 \\
\hline$F$ & 25 & 2.027 & 0.275 & 1.849 & 1.717 & 1.223 & 1.198 \\
\hline$F$ & 35 & 1.672 & 0.137 & 1.085 & 0.859 & 0.521 & 0.513 \\
\hline$F$ & 45 & 1.520 & 0.103 & 0.666 & 0.528 & 0.249 & 0.318 \\
\hline \multirow[t]{2}{*}{$\mathbf{F}$} & \multirow[t]{2}{*}{55} & 1.292 & 0.137 & 0.567 & 0.495 & 0.136 & 0.318 \\
\hline & & $\begin{array}{c}\text { SUM (NON-CL) } \\
\text { LEUKEMIA }\end{array}$ & $\begin{array}{l}\text { KIDNEY \& } \\
\text { BLADDER }\end{array}$ & THYROID & $\begin{array}{l}\text { ALL OTHER } \\
\text { CANCERS }\end{array}$ & & $\begin{array}{c}\text { TOTAL } \\
\text { CANCERS }\end{array}$ \\
\hline$M$ & 25 & 0.860 & 1.797 & 1.084 & 2.767 & & 13.89 \\
\hline M & 35 & 0.935 & 0.985 & 0.705 & 1.234 & & 7.717 \\
\hline M & 45 & 1.009 & 0.638 & 0.466 & 0.673 & & 5.258 \\
\hline M & 55 & 1.009 & 0.464 & 0.277 & 0.523 & & 4.381 \\
\hline $\mathrm{F}$ & 25 & 0.561 & 2.144 & 3.353 & 1.757 & & 16.10 \\
\hline$F$ & 35 & 0.636 & 1.275 & 2.429 & 1.009 & & 10.14 \\
\hline$F$ & 45 & 0.785 & 0.869 & 1.769 & 0.748 & & 7.555 \\
\hline$F$ & 55 & 0.860 & 0.696 & 1.186 & 0.748 & & 6.434 \\
\hline
\end{tabular}

Table 13

NOVEMBER 1960 SPE, EXCESS CANCER MORTALITIES PER 1000 PEOPLE BEHIND $10 \mathrm{~g} / \mathrm{cm}^{* * 2}$ OF AI

\begin{tabular}{|ll|c|c|c|c|c|c|}
\hline \multicolumn{2}{|c|}{ SEX,AGE @ EXP } & LUNG & ESOPHAGUS & STOMACH & COLON & LIVER & PANCREAS \\
\hline $\mathrm{M}$ & 25 & 2.027 & 0.206 & 0.912 & 0.760 & 0.996 & 0.734 \\
$\mathrm{M}$ & 35 & 1.368 & 0.103 & 0.468 & 0.363 & 0.340 & 0.342 \\
$\mathrm{M}$ & 45 & 0.988 & 0.069 & 0.296 & 0.198 & 0.159 & 0.220 \\
$\mathrm{M}$ & 55 & 0.709 & 0.069 & 0.296 & 0.198 & 0.091 & 0.220 \\
$\mathrm{~F}$ & 25 & 1.444 & 0.206 & 1.381 & 0.859 & 1.200 & 1.125 \\
$\mathrm{~F}$ & 35 & 1.216 & 0.103 & 0.814 & 0.429 & 0.498 & 0.489 \\
$\mathrm{~F}$ & 45 & 1.089 & 0.069 & 0.493 & 0.264 & 0.226 & 0.293 \\
$\mathrm{~F}$ & 55 & 0.937 & 0.103 & 0.419 & 0.231 & 0.113 & 0.293 \\
& & & & & & & \\
\cline { 2 - 8 } & & SUM(NON-CL) & KIDNEY \& & THYROID & ALLOTHER & & TOTAL \\
$\mathrm{M}$ & 25 & LEUKEMIA & BLADDER & & CANCERS & & MORTALITIES \\
$\mathrm{nyyyyyyy}$ & 35 & 0.673 & 0.580 & 0.175 & 1.084 & & 8.146 \\
$\mathrm{M}$ & 45 & 0.848 & 0.348 & 0.112 & 0.486 & & 4.678 \\
$\mathrm{M}$ & 55 & 0.823 & 0.232 & 0.073 & 0.262 & & 3.318 \\
$\mathrm{~F}$ & 25 & 0.411 & 0.174 & 0.044 & 0.224 & & 2.847 \\
$F$ & 35 & 0.486 & 0.464 & 0.224 & 0.411 & & 8.396 \\
$\mathrm{~F}$ & 45 & 0.561 & 0.290 & 0.160 & 0.299 & & 5.134 \\
$F$ & 55 & 0.636 & 0.232 & 0.107 & 0.299 & & 3.745 \\
\hline
\end{tabular}


Table 14

AUGUST 1972 SPE, EXCESS CANCERS PER 1000 PEOPLE BEHIND $1 \mathrm{~g} / \mathrm{cm}^{* * 2}$ OF AI

\begin{tabular}{|c|c|c|c|c|c|c|c|}
\hline \multicolumn{2}{|c|}{ SEX,AGE@EXP } & LUNG & ESOPHAGUS & STOMACH & COLON & LIVER & PANCREAS \\
\hline$M$ & 25 & 23.90 & 2.414 & 3.798 & 9.727 & 2.202 & 2.227 \\
\hline$M$ & 35 & 16.09 & 1.034 & 1.935 & 4.421 & 0.718 & 1.044 \\
\hline$M$ & 45 & 11.60 & 0.690 & 1.218 & 2.432 & 0.335 & 0.626 \\
\hline M & 55 & 8.520 & 1.034 & 1.147 & 2.432 & 0.191 & 0.696 \\
\hline$F$ & 25 & 18.93 & 2.759 & 5.375 & 11.50 & 2.585 & 3.410 \\
\hline $\mathrm{F}$ & 35 & 15.62 & 1.379 & 3.153 & 5.748 & 1.101 & 1.462 \\
\hline$F$ & 45 & 14.20 & 1.034 & 1.935 & 3.537 & 0.527 & 0.905 \\
\hline \multirow[t]{2}{*}{$F$} & \multirow[t]{2}{*}{55} & 12.07 & 1.379 & 1.648 & 3.316 & 0.287 & 0.905 \\
\hline & & $\begin{array}{c}\text { SUM (NON-CL) } \\
\text { LEUKEMIA }\end{array}$ & $\begin{array}{l}\text { KIDNEY \& } \\
\text { BLADDER }\end{array}$ & THYROID & $\begin{array}{l}\text { ALL OTHER } \\
\text { CANCERS }\end{array}$ & & $\begin{array}{c}\text { TOTAL } \\
\text { CANCERS }\end{array}$ \\
\hline$M$ & 25 & 10.01 & 7.508 & 19.74 & 32.20 & & 113.7 \\
\hline$M$ & 35 & 10.88 & 4.117 & 12.83 & 14.36 & & 67.43 \\
\hline$M$ & 45 & 11.75 & 2.664 & 8.496 & 7.832 & & 47.64 \\
\hline$M$ & 55 & 11.75 & 1.938 & 5.044 & 6.091 & & 38.84 \\
\hline$F$ & 25 & 6.527 & 8.962 & 61.07 & 20.45 & & 141.56 \\
\hline$F$ & 35 & 7.397 & 5.328 & 44.25 & 11.75 & & 97.19 \\
\hline$F$ & 45 & 9.137 & 3.633 & 32.21 & 8.702 & & 75.82 \\
\hline$F$ & 55 & 10.01 & 2.906 & 21.59 & 8.702 & & 62.82 \\
\hline
\end{tabular}

Table 15

AUGUST 1972 SPE, EXCESS CANCER MORTALITIES PER 1000 PEOPLE BEHIND $1 \mathrm{~g} / \mathrm{cm}^{* *} 2$ OF AI

\begin{tabular}{|ll|c|c|c|c|c|c|}
\hline \multicolumn{2}{|c|}{ SEX, AGE @ EXP } & LUNG & ESOPHAGUS & STOMACH & COLON & LIVER & PANCREAS \\
\hline$M$ & 25 & 18.93 & 2.069 & 2.652 & 5.085 & 2.106 & 2.088 \\
$M$ & 35 & 12.78 & 1.034 & 1.362 & 2.432 & 0.718 & 0.974 \\
$M$ & 45 & 9.229 & 0.690 & 0.860 & 1.326 & 0.335 & 0.626 \\
$M$ & 55 & 6.626 & 0.690 & 0.860 & 1.326 & 0.191 & 0.626 \\
$F$ & 25 & 13.49 & 2.069 & 4.013 & 5.748 & 2.537 & 3.201 \\
$F$ & 35 & 11.36 & 1.034 & 2.365 & 2.874 & 1.053 & 1.392 \\
$F$ & 45 & 10.18 & 0.690 & 1.433 & 1.769 & 0.479 & 0.835 \\
$F$ & 55 & 8.756 & 1.034 & 1.218 & 1.548 & 0.239 & 0.835 \\
& & & & & & & \\
\cline { 2 - 8 } & & SUM (NON-CL) & KIDNEY \& & THYROID & ALL OTHER & & TOTAL \\
\cline { 2 - 8 } M & LEUKEMIA & BLADDER & & CANCERS & & MORTALITIES \\
$M$ & 35 & 7.832 & 2.422 & 3.186 & 12.62 & & 37.15 \\
$M$ & 45 & 8.702 & 1.453 & 2.035 & 5.656 & & 27.98 \\
$M$ & 55 & 9.572 & 0.969 & 1.327 & 3.046 & & 24.03 \\
$F$ & 25 & 4.572 & 0.727 & 0.797 & 2.611 & & 52.84 \\
$F$ & 35 & 5.786 & 3.149 & 5.576 & 8.267 & & 36.53 \\
$F$ & 45 & 6.527 & 1.938 & 4.071 & 4.786 & & 29.52 \\
$F$ & 55 & 7.397 & 0.969 & 1.947 & 3.481 & & 27.42 \\
\hline
\end{tabular}


Table 16

AUGUST 1972 SPE, EXCESS CANCERS PER 1000 PEOPLE BEHIND $10 \mathrm{~g} / \mathrm{cm} * 2$ OF Al

\begin{tabular}{|c|c|c|c|c|c|c|c|}
\hline \multicolumn{2}{|c|}{ SEX,AGE@EXP } & LUNG & ESOPHAGUS & STOMACH & COLON & LIVER & PANCREAS \\
\hline$M$ & 25 & 2.309 & 0.223 & 0.715 & 1.178 & 0.488 & 0.424 \\
\hline$M$ & 35 & 1.554 & 0.095 & 0.364 & 0.536 & 0.159 & 0.199 \\
\hline$M$ & 45 & 1.120 & 0.064 & 0.229 & 0.295 & 0.074 & 0.119 \\
\hline$M$ & 55 & 0.823 & 0.095 & 0.216 & 0.295 & 0.042 & 0.133 \\
\hline$F$ & 25 & 1.829 & 0.254 & 1.012 & 1.393 & 0.573 & 0.649 \\
\hline$F$ & 35 & 1.509 & 0.127 & 0.594 & 0.696 & 0.244 & 0.278 \\
\hline$F$ & 45 & 1.372 & 0.095 & 0.364 & 0.429 & 0.117 & 0.172 \\
\hline \multirow[t]{2}{*}{$F$} & \multirow[t]{2}{*}{55} & 1.166 & 0.127 & 0.310 & 0.402 & 0.064 & 0.172 \\
\hline & & $\begin{array}{c}\text { SUM (NON-CL) } \\
\text { LEUKEMIA }\end{array}$ & $\begin{array}{l}\text { KIDNEY \& } \\
\text { BLADDER }\end{array}$ & THYROID & $\begin{array}{l}\text { ALI OTHER } \\
\text { CANCERS }\end{array}$ & & $\begin{array}{c}\text { TOTAL } \\
\text { CANCERS }\end{array}$ \\
\hline$M$ & 25 & 0.827 & 1.032 & 1.844 & 2.662 & & 11.70 \\
\hline$M$ & 35 & 0.899 & 0.566 & 1.199 & 1.187 & & 6.759 \\
\hline$M$ & 45 & 0.971 & 0.366 & 0.794 & 0.647 & & 4.680 \\
\hline$M$ & 55 & 0.971 & 0.266 & 0.471 & 0.504 & & 3.816 \\
\hline $\mathrm{F}$ & 25 & 0.540 & 1.231 & 5.707 & 1.691 & & 14.88 \\
\hline$F$ & 35 & 0.611 & 0.732 & 4.136 & 0.971 & & 9.898 \\
\hline$F$ & 45 & 0.755 & 0.499 & 3.011 & 0.719 & & 7.533 \\
\hline$F$ & 55 & 0.827 & 0.399 & 2.018 & 0.719 & & 6.205 \\
\hline
\end{tabular}

Table 17

AUGUST 1972 SPE, EXCESS CANCER MORTALITIES PER 1000 PEOPLE BEHIND $10 \mathrm{~g} / \mathrm{cm}^{* *} 2$ OF AI

\begin{tabular}{|ll|l|c|c|c|c|c|}
\hline SEX, AGE @ EXP & LUNG & ESOPHAGUS & STOMACH & COLON & LIVER & PANCREAS \\
\hline $\mathrm{M}$ & 25 & 1.829 & 0.191 & 0.499 & 0.616 & 0.467 & 0.398 \\
$\mathrm{M}$ & 35 & 1.234 & 0.095 & 0.256 & 0.295 & 0.159 & 0.186 \\
$\mathrm{M}$ & 45 & 0.891 & 0.064 & 0.162 & 0.161 & 0.074 & 0.119 \\
$\mathrm{M}$ & 55 & 0.640 & 0.064 & 0.162 & 0.161 & 0.042 & 0.119 \\
$\mathrm{~F}$ & 25 & 1.303 & 0.191 & 0.755 & 0.696 & 0.562 & 0.610 \\
$\mathrm{~F}$ & 35 & 1.097 & 0.095 & 0.445 & 0.348 & 0.233 & 0.265 \\
$\mathrm{~F}$ & 45 & 0.983 & 0.064 & 0.270 & 0.214 & 0.106 & 0.159 \\
$\mathrm{~F}$ & 55 & 0.846 & 0.095 & 0.229 & 0.187 & 0.053 & 0.159 \\
& & & & & & & \\
\cline { 2 - 8 } & & SUM(NON-CL) & KIDNEY \& & THYROID & ALLOTHER & & TOTAL \\
$\mathrm{M}$ & 25 & LEUKEMIA & BLADDER & & CANCERS & & MORTALITIES \\
$\mathrm{M}$ & 35 & 0.647 & 0.333 & 0.298 & 1.043 & & 6.320 \\
$\mathrm{M}$ & 45 & 0.719 & 0.200 & 0.190 & 0.468 & & 3.802 \\
$\mathrm{M}$ & 55 & 0.791 & 0.133 & 0.124 & 0.252 & & 2.771 \\
$\mathrm{~F}$ & 25 & 0.396 & 0.100 & 0.074 & 0.216 & & 2.369 \\
$\mathrm{~F}$ & 35 & 0.468 & 0.433 & 0.521 & 0.683 & & 6.150 \\
$\mathrm{~F}$ & 45 & 0.540 & 0.166 & 0.273 & 0.288 & & 3.994 \\
$\mathrm{~F}$ & 55 & 0.611 & 0.133 & 0.182 & 0.288 & & 3.062 \\
\hline
\end{tabular}


Table 18

OCTOBER 1989 SPE, EXCESS CANCERS PER 1000 PEOPLE BEHIND $1 \mathrm{~g} / \mathrm{cm}^{* * 2}$ OF AI

\begin{tabular}{|c|c|c|c|c|c|c|c|}
\hline \multicolumn{2}{|c|}{ SEX.AGE@EXP } & LUNG & ESOPHAGUS & STOMACH & COLON & LIVER & PANCREAS \\
\hline$M$ & 25 & 11.65 & 1.131 & 2.700 & 5.076 & 1.800 & 1.596 \\
\hline$M$ & 35 & 7.845 & 0.485 & 1.376 & 2.307 & 0.587 & 0.748 \\
\hline$M$ & 45 & 5.653 & 0.323 & 0.866 & 1.269 & 0.274 & 0.449 \\
\hline$M$ & 55 & 4.153 & 0.485 & 0.815 & 1.269 & 0.157 & 0.499 \\
\hline $\mathrm{F}$ & 25 & 9.229 & 1.292 & 3.821 & 5.999 & 2.114 & 2.444 \\
\hline$F$ & 35 & 7.614 & 0.646 & 2.242 & 2.999 & 0.900 & 1.048 \\
\hline$F$ & 45 & 6.922 & 0.485 & 1.376 & 1.846 & 0.431 & 0.648 \\
\hline \multirow[t]{2}{*}{$F$} & \multirow[t]{2}{*}{55} & 5.884 & 0.646 & 1.172 & 1.730 & 0.235 & 0.648 \\
\hline & & $\begin{array}{c}\text { SUM (NON-CL) } \\
\text { LEUKEMIA }\end{array}$ & $\begin{array}{l}\text { KIDNEY \& } \\
\text { BLADDER }\end{array}$ & THYROID & $\begin{array}{l}\text { ALL OTHER } \\
\text { CANCERS }\end{array}$ & & $\begin{array}{c}\text { TOTAL } \\
\text { CANCERS }\end{array}$ \\
\hline M & 25 & 4.562 & 4.632 & 11.72 & 14.68 & & 59.55 \\
\hline M & 35 & 4.959 & 2.540 & 7.622 & 6.545 & & 35.01 \\
\hline M & 45 & 5.355 & 1.644 & 5.047 & 3.570 & & 24.45 \\
\hline M & 55 & 5.355 & 1.195 & 2.996 & 2.777 & & 19.70 \\
\hline$F$ & 25 & 2.975 & 5.529 & 36.27 & 9.322 & & 79.00 \\
\hline$F$ & 35 & 3.372 & 3.287 & 26.28 & 5.355 & & 53.75 \\
\hline$F$ & 45 & 4.165 & 2.241 & 19.14 & 3.967 & & 41.22 \\
\hline$F$ & 55 & 4.562 & 1.793 & 12.83 & 3.967 & & 33.46 \\
\hline
\end{tabular}

Table 19

OCTOBER 1989 SPE, EXCESS CANCER MORTALITIES PER 1000 PEOPLE BEHIND $1 \mathrm{~g} / \mathrm{cm}^{*} 2$ OF AI

\begin{tabular}{|ll|c|c|c|c|c|c|}
\hline \multicolumn{2}{|c|}{ SEX, AGE @ EXP } & LUNG & ESOPHAGUS & STOMACH & COLON & LIVER & PANCREAS \\
\hline $\mathrm{M}$ & 25 & 9.229 & 0.969 & 1.885 & 2.653 & 1.722 & 1.496 \\
$\mathrm{M}$ & 35 & 6.230 & 0.485 & 0.968 & 1.269 & 0.587 & 0.698 \\
$\mathrm{M}$ & $4 J$ & 4.499 & 0.323 & 0.611 & 0.692 & 0.274 & 0.449 \\
$\mathrm{M}$ & 55 & 3.230 & 0.323 & 0.611 & 0.692 & 0.157 & 0.449 \\
$\mathrm{~F}$ & 25 & 6.576 & 0.969 & 2.853 & 2.999 & 2.074 & 2.295 \\
$\mathrm{~F}$ & 35 & 5.537 & 0.485 & 1.681 & 1.500 & 0.861 & 0.998 \\
$\mathrm{~F}$ & 45 & 4.961 & 0.323 & 1.019 & 0.923 & 0.391 & 0.599 \\
$\mathrm{~F}$ & 55 & 4.268 & 0.485 & 0.866 & 0.808 & 0.196 & 0.599 \\
& & & & & & & \\
\cline { 2 - 8 } & & SUM(NON-CL) & KIDNEY\& & THYROID & ALLOTHER & & TOTAL \\
\cline { 2 - 8 } $\mathrm{M}$ & 25 & 3.570 & 1.494 & 1.892 & 5.752 & & 30.66 \\
$\mathrm{M}$ & 35 & 3.967 & 0.897 & 1.209 & 2.578 & & 18.89 \\
$\mathrm{M}$ & 45 & 4.364 & 0.598 & 0.789 & 1.388 & & 13.99 \\
$\mathrm{M}$ & 55 & 4.364 & 0.448 & 0.473 & 1.190 & & 11.94 \\
$\mathrm{~F}$ & 25 & 2.182 & 1.943 & 3.312 & 3.769 & & 28.97 \\
$\mathrm{~F}$ & 35 & 2.578 & 1.195 & 2.418 & 2.182 & & 19.44 \\
$\mathrm{~F}$ & 45 & 2.975 & 0.747 & 1.735 & 1.587 & & 15.26 \\
$\mathrm{~F}$ & 55 & 3.372 & 0.598 & 1.156 & 1.587 & & 13.93 \\
\hline
\end{tabular}


Table 20

OCTOBER 1989 SPE, EXCESS CANCERS PER 1000 PEOPLE BEHIND $10 \mathrm{~g} / \mathrm{cm}^{* *} 2$ OF AI

\begin{tabular}{|c|c|c|c|c|c|c|c|}
\hline \multicolumn{2}{|c|}{ SEX,AGE@EXP } & LUNG & ESOPHAGUS & STOMACH & COLON & LIVER & PANCREAS \\
\hline$M$ & 25 & 2.116 & 0.199 & 0.855 & 1.135 & 0.640 & 0.510 \\
\hline$M$ & 35 & 1.424 & 0.085 & 0.436 & 0.516 & 0.209 & 0.239 \\
\hline$M$ & 45 & 1.026 & 0.057 & 0.274 & 0.284 & 0.097 & 0.143 \\
\hline$M$ & 55 & 0.754 & 0.085 & 0.258 & 0.284 & 0.056 & 0.159 \\
\hline$F$ & 25 & 1.676 & 0.228 & 1.210 & 1.342 & 0.752 & 0.781 \\
\hline $\mathrm{F}$ & 35 & 1.382 & 0.114 & 0.710 & 0.671 & 0.320 & 0.335 \\
\hline$F$ & 45 & 1.257 & 0.085 & 0.436 & 0.413 & 0.153 & 0.207 \\
\hline \multirow[t]{2}{*}{$F$} & \multirow[t]{2}{*}{55} & 1.068 & 0.114 & 0.371 & 0.387 & 0.084 & 0.207 \\
\hline & & $\begin{array}{c}\text { SUM (NON-CL) } \\
\text { LEUKEMIA }\end{array}$ & $\begin{array}{l}\text { KIDNEY \& } \\
\text { BLADDER } \\
\end{array}$ & THYROID & $\begin{array}{c}\text { ALL OTHER } \\
\text { CANCERS }\end{array}$ & & $\begin{array}{c}\text { TOTAL } \\
\text { CANCERS }\end{array}$ \\
\hline$M$ & 25 & 0.727 & 1.177 & 1.282 & 2.338 & & 10.98 \\
\hline$M$ & 35 & 0.790 & 0.646 & 0.833 & 1.043 & & 6.220 \\
\hline$M$ & 45 & 0.853 & 0.418 & 0.552 & 0.569 & & 4.273 \\
\hline$M$ & 55 & 0.853 & 0.304 & 0.328 & 0.442 & & 3.523 \\
\hline$F$ & 25 & 0.474 & 1.405 & 3.966 & 1.485 & & 13.32 \\
\hline$F$ & 35 & 0.537 & 0.835 & 2.874 & 0.853 & & 8.631 \\
\hline$F$ & 45 & 0.663 & 0.570 & 2.092 & 0.632 & & 6.508 \\
\hline$F$ & 55 & 0.727 & 0.456 & 1.402 & 0.632 & & 5.447 \\
\hline
\end{tabular}

Table 21

OCTOBER 1989 SPE, EXCESS CANCER MORTALITIES PER 1000 PEOPLE BEHIND $10 \mathrm{~g} / \mathrm{cm}^{* * 2}$ OF AI

\begin{tabular}{|ll|c|c|c|c|c|c|}
\hline SEX, AGE @ EXP & LUNG & ESOPHAGUS & STOMACH & COLON & LIVER & PANOREAS \\
\hline$M$ & 25 & 1.676 & 0.171 & 0.597 & 0.593 & 0.612 & 0.478 \\
$M$ & 35 & 1.131 & 0.085 & 0.306 & 0.284 & 0.209 & 0.223 \\
$M$ & 45 & 0.817 & 0.057 & 0.194 & 0.155 & 0.097 & 0.143 \\
$M$ & 55 & 0.586 & 0.057 & 0.194 & 0.155 & 0.056 & 0.143 \\
$F$ & 25 & 1.194 & 0.171 & 0.903 & 0.671 & 0.738 & 0.733 \\
$F$ & 35 & 1.005 & 0.085 & 0.532 & 0.335 & 0.306 & 0.319 \\
$F$ & 45 & 0.901 & 0.057 & 0.323 & 0.206 & 0.139 & 0.191 \\
$F$ & 55 & 0.775 & 0.085 & 0.274 & 0.181 & 0.070 & 0.191 \\
& & & & & & & TOTAL \\
\cline { 2 - 8 } & & SUM(NON-CL) & KIDNEY \& & THYROID & ALLOTHER & & MORTALITIES \\
\cline { 2 - 8 }$M$ & 25 & LEUKEMIA & BLADDER & & CANCERS & & 6.199 \\
$M$ & 35 & 0.569 & 0.380 & 0.207 & 0.916 & & 3.641 \\
$M$ & 45 & 0.632 & 0.228 & 0.132 & 0.411 & & 2.617 \\
$M$ & 55 & 0.695 & 0.152 & 0.086 & 0.221 & & 2.241 \\
$F$ & 25 & 0.348 & 0.114 & 0.052 & 0.190 & & 6.213 \\
$F$ & 35 & 0.411 & 0.304 & 0.264 & 0.348 & & 3.910 \\
$F$ & 45 & 0.474 & 0.190 & 0.190 & 0.253 & & 2.923 \\
$F$ & 55 & 0.537 & 0.152 & 0.126 & 0.253 & & 2.644 \\
\hline
\end{tabular}


Table 22

AUG., SEP., \& OCT. 1989 SPE'S, EXCESS CANCERS PER 1000 PEOPLE

BEHIND $1 \mathrm{~g} / \mathrm{cm}^{* * 2}$ OF AI

\begin{tabular}{|c|c|c|c|c|c|c|c|}
\hline \multicolumn{2}{|c|}{ SEX,AGE@ EXP } & LUNG & ESOPHAGUS & STOMACH & COLON & LIVER & PANCREAS \\
\hline $\bar{M}$ & 25 & 21.88 & 2.174 & 4.636 & 9.337 & 3.029 & 2.735 \\
\hline$M$ & 35 & 14.73 & 0.932 & 2.362 & 4.244 & 0.988 & 1.282 \\
\hline$M$ & 45 & 10.62 & 0.621 & 1.487 & 2.334 & 0.461 & 0.769 \\
\hline M & 55 & 7.800 & 0.932 & 1.400 & 2.334 & 0.263 & 0.855 \\
\hline$F$ & 25 & 17.33 & 2.484 & 6.560 & 11.04 & 3.556 & 4.189 \\
\hline$F$ & 35 & 14.30 & 1.242 & 3.849 & 5.517 & 1.515 & 1.795 \\
\hline$F$ & 45 & 13.00 & 0.932 & 2.362 & 3.395 & 0.724 & 1.111 \\
\hline \multirow[t]{2}{*}{$F$} & 55 & 11.05 & 1.242 & 2.012 & 3.183 & 0.395 & 1.111 \\
\hline & & $\begin{array}{c}\text { SUM (NON-CL) } \\
\text { LEUKEMIA }\end{array}$ & $\begin{array}{l}\text { KIDNEY \& } \\
\text { BLADDER }\end{array}$ & THYROID & $\begin{array}{c}\text { ALL OTHER } \\
\text { CANCERS }\end{array}$ & & $\begin{array}{c}\text { TOTAL } \\
\text { CANCERS }\end{array}$ \\
\hline M & 25 & 8.986 & 8.638 & 19.51 & 28.91 & & 109.8 \\
\hline M & 35 & 9.768 & 4.737 & 12.68 & 12.89 & & 64.62 \\
\hline M & 45 & 10.55 & 3.065 & 8.397 & 7.033 & & 45.33 \\
\hline M & 55 & 10.55 & 2.229 & 4.986 & 5.470 & & 36.82 \\
\hline$F$ & 25 & 5.861 & 10.31 & 60.35 & 18.36 & & 140.0 \\
\hline$F$ & 35 & 6.642 & 6.130 & 43.73 & 10.55 & & 95.27 \\
\hline$F$ & 45 & 8.205 & 4.180 & 31.84 & 7.814 & & 73.56 \\
\hline$F$ & 55 & 8.986 & 3.344 & 21.34 & 7.814 & & 60.48 \\
\hline
\end{tabular}

Table 23

AUG., SEP., \& OCT. 1989 SPE'S, EXCESS CANCER MORTALITIES PER 1000 PEOPLE

BEHIND $1 \mathrm{~g} / \mathrm{cm}^{*} 2$ OF AI

\begin{tabular}{|c|c|c|c|c|c|c|c|}
\hline & @EXP & LUNG & ESOPHAGUS & STOMACH & COLON & LIVER & PANCREAS \\
\hline$M$ & 25 & 17.33 & 1.863 & 3.237 & 4.881 & 2.898 & 2.564 \\
\hline$M$ & 35 & 11.70 & 0.932 & 1.662 & 2.334 & 0.988 & 1.197 \\
\hline$M$ & 45 & 8.450 & 0.621 & 1.050 & 1.273 & 0.461 & 0.769 \\
\hline$M$ & 55 & 6.067 & 0.621 & 1.050 & 1.273 & 0.263 & 0.769 \\
\hline $\mathrm{F}$ & 25 & 12.35 & 1.863 & 4.898 & 5.517 & 3.490 & 3.932 \\
\hline $\mathrm{F}$ & 35 & 10.40 & 0.932 & 2.887 & 2.759 & 1.449 & 1.710 \\
\hline$F$ & 45 & 9.317 & 0.621 & 1.749 & 1.698 & 0.659 & 1.026 \\
\hline$F$ & 55 & 8.017 & 0.932 & 1.487 & 1.485 & 0.329 & 1.026 \\
\hline & & $\begin{array}{c}\text { SUM (NON-CL) } \\
\text { LEUKEMIA }\end{array}$ & $\begin{array}{l}\text { KIDNEY \& } \\
\text { BLADDER }\end{array}$ & THYROID & $\begin{array}{l}\text { AL OTHER } \\
\text { CANCERS }\end{array}$ & & $\begin{array}{c}\text { TOTAL } \\
\text { MORTALITIES }\end{array}$ \\
\hline$M$ & 25 & 7.033 & 2.787 & 3.149 & 11.33 & & 57.07 \\
\hline$M$ & 35 & 7.814 & 1.672 & 2.012 & 5.079 & & 35.39 \\
\hline$M$ & 45 & 8.595 & 1.115 & 1.312 & 2.735 & & 26.38 \\
\hline$M$ & 55 & 8.595 & 0.836 & 0.787 & 2.344 & & 22.61 \\
\hline$F$ & 25 & 4.298 & 3.623 & 5.510 & 7.423 & & 52.91 \\
\hline$F$ & 35 & 5.079 & 2.229 & 4.024 & 4.298 & & 35.77 \\
\hline$F$ & 45 & 5.861 & 1.393 & 2.886 & 3.126 & & 28.34 \\
\hline$F$ & 55 & 6.642 & 1.115 & 1.924 & 3.126 & & 26.08 \\
\hline
\end{tabular}


Table 24

AUG., SEP., \& OCT. 1989 SPE'S, EXCESS CANCERS PER 1000 PEOPLE

BEHIND $10 \mathrm{~g} / \mathrm{cm}^{* * 2}$ OF AI

\begin{tabular}{|c|c|c|c|c|c|c|c|}
\hline \multicolumn{2}{|c|}{ SEX.AGE@EXP } & UNG & ESOPHAGUS & STOMACH & COLON & LIVER & PANCREAS \\
\hline$M$ & 25 & 3.431 & 0.329 & 1.377 & 1.864 & 1.026 & 0.821 \\
\hline$M$ & 35 & 2.310 & 0.141 & 0.701 & 0.847 & 0.335 & 0.385 \\
\hline$M$ & 45 & 1.664 & 0.094 & 0.442 & 0.466 & 0.156 & 0.231 \\
\hline$M$ & 55 & 1.223 & 0.141 & 0.416 & 0.466 & 0.089 & 0.256 \\
\hline$F$ & 25 & 2.717 & 0.377 & 1.948 & 2.203 & 1.205 & 1.257 \\
\hline $\mathrm{F}$ & 35 & 2.242 & 0.188 & 1.143 & 1.102 & 0.513 & 0.539 \\
\hline$F$ & 45 & 2.038 & 0.141 & 0.701 & 0.678 & 0.245 & 0.333 \\
\hline \multirow[t]{2}{*}{$F$} & \multirow[t]{2}{*}{55} & 1.732 & 0.188 & 0.598 & 0.636 & 0.134 & 0.333 \\
\hline & & $\begin{array}{c}\text { SUM (NON-CL) } \\
\text { LEUKEMIA }\end{array}$ & $\begin{array}{l}\text { KIDNEY \& } \\
\text { BLADDER }\end{array}$ & THYROID & $\begin{array}{l}\text { ALL OTHER } \\
\text { CANCERS }\end{array}$ & & $\begin{array}{c}\text { TOTAL } \\
\text { CANCERS }\end{array}$ \\
\hline$M$ & 25 & 1.208 & 1.987 & 1.944 & 3.888 & & 17.88 \\
\hline$M$ & 35 & 1.313 & 1.090 & 1.264 & 1.734 & & 10.12 \\
\hline$M$ & 45 & 1.419 & 0.705 & 0.837 & 0.946 & & 6.959 \\
\hline$M$ & 55 & 1.419 & 0.513 & 0.497 & 0.736 & & 5.755 \\
\hline$F$ & 25 & 0.788 & 2.372 & 6.014 & 2.469 & & 21.35 \\
\hline$F$ & 35 & 0.893 & 1.410 & 4.358 & 1.419 & & 13.81 \\
\hline$F$ & 45 & 1.103 & 0.961 & 3.173 & 1.051 & & 10.43 \\
\hline $\mathrm{F}$ & 55 & 1.208 & 0.769 & 2.127 & 1.051 & & 8.776 \\
\hline
\end{tabular}

Table 25

AUG., SEP., \& OCT. 1989 SPE'S, EXCESS CANCER MORTALITIES PER 1000 PEOPLE

BEHIND $10 \mathrm{~g} / \mathrm{cm}^{* * 2}$ OF AI

\begin{tabular}{|c|c|c|c|c|c|c|c|}
\hline \multicolumn{2}{|c|}{ SEX,AGE@EXP } & LUNG & ESOPHAGUS & STOMACH & COLON & LIVER & PANCREAS \\
\hline$M$ & 25 & 2.717 & 0.282 & 0.961 & 0.975 & 0.982 & 0.769 \\
\hline$M$ & 35 & 1.834 & 0.141 & 0.494 & 0.466 & 0.335 & 0.359 \\
\hline$M$ & 45 & 1.325 & 0.094 & 0.312 & 0.254 & 0.156 & 0.231 \\
\hline$M$ & 55 & 0.951 & 0.094 & 0.312 & 0.254 & 0.089 & 0.231 \\
\hline $\mathbf{F}$ & 25 & 1.936 & 0.282 & 1.455 & 1.102 & 1.182 & 1.180 \\
\hline$F$ & 35 & 1.630 & 0.141 & 0.857 & 0.551 & 0.491 & 0.513 \\
\hline$F$ & 45 & 1.461 & 0.094 & 0.520 & 0.339 & 0.223 & 0.308 \\
\hline \multirow[t]{2}{*}{$F$} & \multirow[t]{2}{*}{55} & 1.257 & 0.141 & 0.442 & 0.297 & 0.112 & 0.308 \\
\hline & & $\begin{array}{l}\text { SUM (NON-CL) } \\
\text { LEUKEMIA }\end{array}$ & $\begin{array}{l}\text { KIONEY \& } \\
\text { BLADDER }\end{array}$ & THYROID & $\begin{array}{c}\text { ALL OTHER } \\
\text { CANCERS }\end{array}$ & & $\begin{array}{c}\text { TOTAL } \\
\text { MORTALITIES }\end{array}$ \\
\hline$M$ & 25 & 0.946 & 0.641 & 0.314 & 1.524 & & 10.11 \\
\hline$M$ & 35 & 1.051 & 0.385 & 0.200 & 0.683 & & 5.948 \\
\hline$M$ & 45 & 1.156 & 0.256 & 0.131 & 0.368 & & 4.283 \\
\hline$M$ & 55 & 1.156 & 0.192 & 0.078 & 0.315 & & 3.673 \\
\hline$F$ & 25 & 0.578 & 0.833 & 0.549 & 0.998 & & 10.10 \\
\hline$F$ & 35 & 0.683 & 0.513 & 0.401 & 0.578 & & 6.358 \\
\hline$F$ & 45 & 0.788 & 0.320 & 0.288 & 0.420 & & 4.761 \\
\hline$F$ & 55 & 0.893 & 0.256 & 0.192 & 0.420 & & 4.317 \\
\hline
\end{tabular}


Table 26

Excess Cancers per 100025 yr. old Males Behind $1 \mathrm{~g} / \mathrm{cm}^{* * 2}$ of Al (Aug. 72 Flare, Rem data)

\begin{tabular}{l|c|c|c} 
Organ & Primary Protons & Secondaries \& Recoils & Total \\
\hline Lung & 22 & 2 & 24 \\
Colon & 8 & 2 & 10 \\
Kidney \& Bladder & 6 & 2 & 8 \\
Total Cancers & 98 & 16 & 114 \\
\hline
\end{tabular}

Table 27

Excess Cancer Mortalities per 100025 yr. old Males Behind $1 \mathrm{~g} / \mathrm{cm}^{* * 2}$ of Al (Aug. 72 Flare, Rem data)

\begin{tabular}{l|c|c|c} 
Organ & Primary Protons & Secondaries \& Recoils & Total \\
\hline Lung & 18 & 1 & 19 \\
Colon & 4 & 1 & 5 \\
Kidney \& Bladder & 2 & 0.5 & 2.5 \\
Total Cancers & 51 & 8 & 59 \\
\hline
\end{tabular}

Table 28

Excess Cancers per $100025 \mathrm{yr}$. old Males Behind $10 \mathrm{~g} / \mathrm{cm}^{* * 2}$ of Al (Aug. 72 Flare, Rem data)

\begin{tabular}{|c|c|c|c|}
\hline Organ & Primary Protons & Secondaries \& Recoils & Total \\
\hline Lung & 2.1 & 0.2 & 2.3 \\
\hline Colon & 0.9 & 0.3 & 1.2 \\
\hline Kidney \& Bladder & 0.8 & 0.2 & 1 \\
\hline Total Cancers & 10 & 2 & 12 \\
\hline
\end{tabular}

Table 29

Excess Cancer Mortalities per $100025 \mathrm{yr}$. old Males Behind $10 \mathrm{~g} / \mathrm{cm}^{* * 2} 2$ of Al (Aug. 72 Flare, Rem data)

\begin{tabular}{l|c|c|c} 
Organ & Primary Protons & \multicolumn{1}{c}{ Secondaries \& Recoils } & Total \\
\hline Lung & 1.7 & 0.1 & 1.8 \\
Colon & 0.5 & 0.1 & 0.6 \\
Kidney \& Bladder & 0.3 & 0.05 & 0.35 \\
Total Cancers & 5 & 1 & 6 \\
\hline
\end{tabular}



Table 31

EXCESS CANCERS PER 1000 PEOPLE AT STS DLOC 1

\begin{tabular}{|c|c|c|c|c|c|c|c|}
\hline \multicolumn{2}{|c|}{ SEX,AGE@EXP } & LUNG & ESOPHAGUS & STOMACH & COLON & LIVER & PANCREAS \\
\hline$M$ & 25 & 5.469 & 0.382 & 0.930 & 1.797 & 0.596 & 0.550 \\
\hline$M$ & 35 & 3.682 & 0.164 & 0.474 & 0.817 & 0.194 & 0.258 \\
\hline$M$ & 45 & 2.653 & 0.109 & 0.298 & 0.449 & 0.091 & 0.155 \\
\hline$M$ & 55 & 1.949 & 0.164 & 0.281 & 0.449 & 0.052 & 0.172 \\
\hline$F$ & 25 & 4.332 & 0.436 & 1.316 & 2.124 & 0.700 & 0.842 \\
\hline$F$ & 35 & 3.574 & 0.218 & 0.772 & 1.062 & 0.298 & 0.361 \\
\hline$F$ & 45 & 3.249 & 0.164 & 0.474 & 0.653 & 0.143 & 0.223 \\
\hline \multirow[t]{2}{*}{$F$} & \multirow[t]{2}{*}{55} & 2.762 & 0.218 & 0.404 & 0.613 & 0.078 & 0.223 \\
\hline & & $\begin{array}{c}\text { SUM (NON-CL) } \\
\text { LEUKEMIA }\end{array}$ & $\begin{array}{l}\text { KIDNEY \& } \\
\text { BLADDER }\end{array}$ & THYROID & $\begin{array}{c}\text { ALL OTHER } \\
\text { CANCERS }\end{array}$ & & $\begin{array}{c}\text { TOTAL } \\
\text { CANCERS }\end{array}$ \\
\hline$M$ & 25 & 1.477 & 1.450 & 4.287 & 4.753 & & 21.69 \\
\hline$M$ & 35 & 1.606 & 0.795 & 2.787 & 2.120 & & 12.90 \\
\hline$M$ & 45 & 1.734 & 0.514 & 1.845 & 1.156 & & 9.006 \\
\hline$M$ & 55 & 1.734 & 0.374 & 1.096 & 0.899 & & 7.170 \\
\hline$F$ & 25 & 0.964 & 1.730 & 13.26 & 3.019 & & 28.73 \\
\hline$F$ & 35 & 1.092 & 1.029 & 9.611 & 1.734 & & 19.75 \\
\hline$F$ & 45 & 1.349 & 0.701 & 6.997 & 1.285 & & 15.24 \\
\hline$F$ & 55 & 1.477 & 0.561 & 4.690 & 1.285 & & 12.31 \\
\hline
\end{tabular}

Table 32

EXCESS CANCER MORTALITIES PER 1000 PEOPLE AT STS DLOC 1

\begin{tabular}{|ll|c|c|c|c|c|c|}
\hline SEX,AGE@ EXP & LUNG & ESOPHAGUS & STOMACH & COLON & LIVER & PANCREAS \\
\hline$M$ & 25 & 4.332 & 0.327 & 0.649 & 0.939 & 0.570 & 0.515 \\
$M$ & 35 & 2.924 & 0.164 & 0.333 & 0.449 & 0.194 & 0.240 \\
$M$ & 45 & 2.112 & 0.109 & 0.211 & 0.245 & 0.091 & 0.155 \\
$M$ & 55 & 1.516 & 0.109 & 0.211 & 0.245 & 0.052 & 0.155 \\
$F$ & 25 & 3.087 & 0.327 & 0.983 & 1.062 & 0.687 & 0.790 \\
$F$ & 35 & 2.599 & 0.164 & 0.579 & 0.531 & 0.285 & 0.343 \\
$F$ & 45 & 2.328 & 0.109 & 0.351 & 0.327 & 0.130 & 0.206 \\
$F$ & 55 & 2.004 & 0.164 & 0.298 & 0.286 & 0.065 & 0.206 \\
& & & & & & & \\
\cline { 2 - 8 } & & SUM (NON-CL) & KIDNEY \& & THYROID & ALLOTHER & & TOTAL \\
$M$ & 25 & LEUKEMIA & BLADDER & & CANCERS & & MORTALITIES \\
\cline { 2 - 8 } & 1.156 & 0.468 & 0.692 & 1.863 & & 11.51 \\
$M$ & 35 & 1.285 & 0.281 & 0.442 & 0.835 & & 7.148 \\
$M$ & 45 & 1.413 & 0.187 & 0.288 & 0.450 & & 5.260 \\
$M$ & 55 & 1.413 & 0.140 & 0.173 & 0.385 & & 4.399 \\
$F$ & 25 & 0.707 & 0.608 & 1.211 & 1.220 & & 10.68 \\
$F$ & 35 & 0.835 & 0.374 & 0.884 & 0.707 & & 7.301 \\
$F$ & 45 & 0.964 & 0.234 & 0.634 & 0.514 & & 5.796 \\
$F$ & 55 & 1.092 & 0.187 & 0.423 & 0.514 & & 5.238 \\
\hline
\end{tabular}


Table 33

EXCESS CANCERS PER 1000 PEOPLE AT STS DLOC 2

\begin{tabular}{|c|c|c|c|c|c|c|c|}
\hline \multicolumn{2}{|c|}{ SEX,AGE@EXP } & LUNG & ESOPHAGUS & STOMACH & COLON & LIVER & PANCREAS \\
\hline$M$ & 25 & 12.60 & 0.893 & 1.819 & 3.958 & 1.117 & 1.071 \\
\hline$M$ & 35 & 8.485 & 0.383 & 0.927 & 1.799 & 0.364 & 0.502 \\
\hline$M$ & 45 & 6.114 & 0.255 & 0.583 & 0.990 & 0.170 & 0.301 \\
\hline$M$ & 55 & 4.492 & 0.383 & 0.549 & 0.990 & 0.097 & 0.335 \\
\hline $\mathrm{F}$ & 25 & 9.982 & 1.020 & 2.574 & 4.678 & 1.311 & 1.640 \\
\hline$F$ & 35 & 8.235 & 0.510 & 1.510 & 2.339 & 0.559 & 0.703 \\
\hline$F$ & 45 & 7.486 & 0.383 & 0.927 & 1.439 & 0.267 & 0.435 \\
\hline \multirow[t]{2}{*}{$F$} & 55 & 6.363 & 0.510 & 0.789 & 1.349 & 0.146 & 0.435 \\
\hline & & $\begin{array}{c}\text { SUM (NON-CL) } \\
\text { LEUKEMIA }\end{array}$ & $\begin{array}{l}\text { KIDNEY \& } \\
\text { BLADDER }\end{array}$ & THYROID & $\begin{array}{c}\text { ALL OTHER } \\
\text { CANCERS }\end{array}$ & & $\begin{array}{l}\text { TOTAL } \\
\text { CANCERS } \\
\end{array}$ \\
\hline M & 25 & 3.552 & 3.136 & 8.707 & 11.43 & & 48.29 \\
\hline M & 35 & 3.861 & 1.720 & 5.661 & 5.097 & & 28.80 \\
\hline M & 45 & 4.170 & 1.113 & 3.748 & 2.780 & & 20.23 \\
\hline M & 55 & 4.170 & 0.809 & 2.226 & 2.162 & & 16.21 \\
\hline$F$ & 25 & 2.317 & 3.743 & 26.94 & 7.259 & & 61.47 \\
\hline$F$ & 35 & 2.626 & 2.226 & 19.52 & 4.170 & & 42.40 \\
\hline$F$ & 45 & 3.244 & 1.518 & 14.21 & 3.089 & & 33.00 \\
\hline$F$ & 55 & 3.552 & 1.214 & 9.527 & 3.089 & & 26.98 \\
\hline
\end{tabular}

Table 34

EXCESS CANCER MORTALITIES PER 1000 PEOPLE AT STS DLOC 2

\begin{tabular}{|c|c|c|c|c|c|c|c|}
\hline \multicolumn{2}{|c|}{ SEX,AGE@EXP } & LUNG & ESOPHAGUS & STOMACH & COLON & LIVER & PANCREAS \\
\hline$M$ & 25 & 9.982 & 0.765 & 1.270 & 2.069 & 1.069 & 1.004 \\
\hline$M$ & 35 & 6.738 & 0.383 & 0.652 & 0.990 & 0.364 & 0.469 \\
\hline 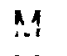 & 45 & 4.866 & 0.255 & 0.412 & 0.540 & 0.170 & 0.301 \\
\hline$M$ & 55 & 3.494 & 0.255 & 0.412 & 0.540 & 0.097 & 0.301 \\
\hline$F$ & 25 & 7.112 & 0.765 & 1.922 & 2.339 & 1.287 & 1.540 \\
\hline$F$ & 35 & 5.989 & 0.383 & 1.132 & 1.169 & 0.534 & 0.669 \\
\hline$F$ & 45 & 5.365 & 0.255 & 0.686 & 0.720 & 0.243 & 0.402 \\
\hline \multirow[t]{2}{*}{$F$} & 55 & 4.617 & 0.383 & 0.583 & 0.630 & 0.121 & 0.402 \\
\hline & & $\begin{array}{c}\text { SUM (NON-CL) } \\
\text { LEUKEMIA }\end{array}$ & $\begin{array}{l}\text { KIDNEY \& } \\
\text { BLADDER }\end{array}$ & THYROID & $\begin{array}{l}\text { ALL OTHER } \\
\text { CANCERS }\end{array}$ & & $\begin{array}{c}\text { TOTAL } \\
\text { MORTALITIES }\end{array}$ \\
\hline$M$ & 25 & 2.780 & 1.012 & 1.406 & 4.479 & & 25.84 \\
\hline$M$ & 35 & 3.089 & 0.607 & 0.898 & 2.008 & & 16.20 \\
\hline$M$ & 45 & 3.398 & 0.405 & 0.586 & 1.081 & & 12.01 \\
\hline$M$ & 55 & 3.398 & 0.304 & 0.351 & 0.927 & & 10.08 \\
\hline$F$ & 25 & 1.699 & 1.315 & 2.460 & 2.935 & & 23.37 \\
\hline$F$ & 35 & 2.008 & 0.809 & 1.796 & 1.699 & & 16.19 \\
\hline$F$ & 45 & 2.317 & 0.506 & 1.288 & 1.236 & & 13.02 \\
\hline$F$ & 55 & 2.626 & 0.405 & 0.859 & 1.236 & & 11.86 \\
\hline
\end{tabular}




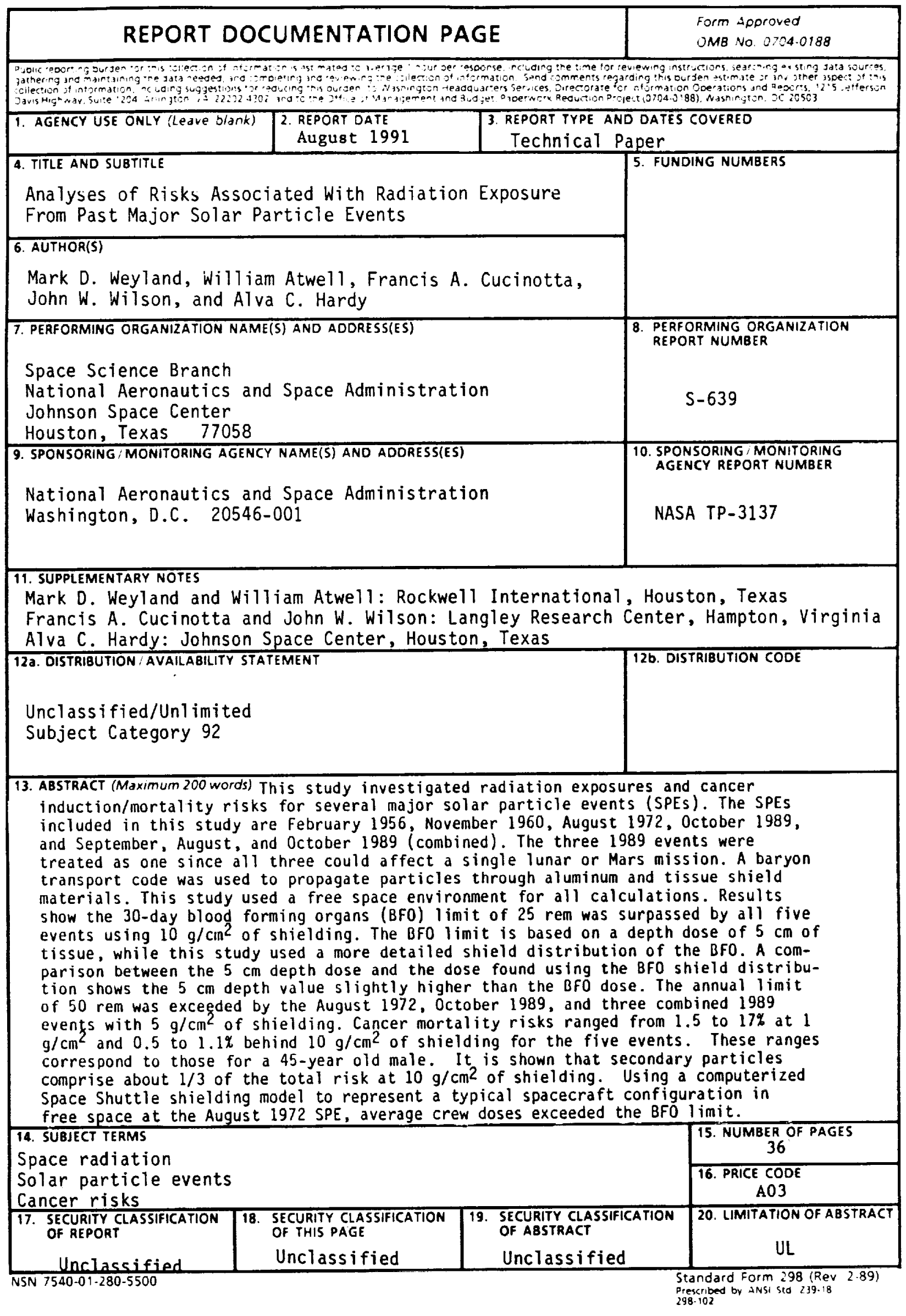


